\title{
Low rank subspace clustering (LRSC)
}

\author{
René Vidal $^{\mathrm{a}, *}$, Paolo Favaro ${ }^{\mathrm{b}}$ \\ ${ }^{a}$ Center for Imaging Science, Department of Biomedical Engineering, Johns Hopkins University, Baltimore, MD 21218, USA \\ ${ }^{\mathrm{b}}$ Institute of Informatics and Applied Mathematics, University of Bern, 3012, Switzerland
}

\section{A R T I C L E I N F O}

\section{Article history:}

Available online $\mathrm{xxxx}$

Communicated by Kim Boyer

\section{Keywords:}

Subspace clustering

Low-rank and sparse methods

Principal component analysis

Motion segmentation

Face clustering

\begin{abstract}
A B S T R A C T
We consider the problem of fitting a union of subspaces to a collection of data points drawn from one or more subspaces and corrupted by noise and/or gross errors. We pose this problem as a non-convex optimization problem, where the goal is to decompose the corrupted data matrix as the sum of a clean and self-expressive dictionary plus a matrix of noise and/or gross errors. By self-expressive we mean a dictionary whose atoms can be expressed as linear combinations of themselves with low-rank coefficients. In the case of noisy data, our key contribution is to show that this non-convex matrix decomposition problem can be solved in closed form from the SVD of the noisy data matrix. The solution involves a novel polynomial thresholding operator on the singular values of the data matrix, which requires minimal shrinkage. For one subspace, a particular case of our framework leads to classical PCA, which requires no shrinkage. For multiple subspaces, the low-rank coefficients obtained by our framework can be used to construct a data affinity matrix from which the clustering of the data according to the subspaces can be obtained by spectral clustering. In the case of data corrupted by gross errors, we solve the problem using an alternating minimization approach, which combines our polynomial thresholding operator with the more traditional shrinkage-thresholding operator. Experiments on motion segmentation and face clustering show that our framework performs on par with state-of-the-art techniques at a reduced computational cost.
\end{abstract}

(c) 2013 Elsevier B.V. All rights reserved.

\section{Introduction}

The past few decades have seen an explosion in the availability of datasets from multiple modalities. While such datasets are usually very high-dimensional, their intrinsic dimension is often much smaller than the dimension of the ambient space. For instance, the number of pixels in an image can be huge, yet most computer vision models use a few parameters to describe the appearance, geometry and dynamics of a scene. This has motivated the development of a number of techniques for finding low-dimensional representations of high-dimensional data.

One of the most commonly used methods is Principal Component Analysis (PCA), which models the data with a single lowdimensional subspace. In practice, however, the data points could be drawn from multiple subspaces and the membership of the data points to the subspaces could be unknown. For instance, a video sequence could contain several moving objects and different subspaces might be needed to describe the motion of different objects in the scene. Therefore, there is a need to simultaneously cluster the data into multiple subspaces and find a low-dimensional subspace fitting each group of points. This problem, known

\footnotetext{
* Corresponding author. Tel.: +1 410516 7306; fax: +1 4105167775.

E-mail address: rvidal@cis.jhu.edu (R. Vidal).
}

as subspace clustering, finds numerous applications in computer vision, e.g., image segmentation (Yang et al., 2008), motion segmentation (Vidal et al., 2008) and face clustering (Ho et al., 2003), image processing, e.g., image representation and compression (Hong et al., 2006), and systems theory, e.g., hybrid system identification (Vidal et al., 2003b).

\subsection{Prior work on subspace clustering}

Over the past decade, a number of subspace clustering methods have been developed. This includes algebraic methods (Boult and Brown, 1991; Costeira and Kanade, 1998; Gear, 1998; Vidal et al., 2003a; Vidal et al., 2004; Vidal et al., 2005), iterative methods (Bradley and Mangasarian, 2000; Tseng, 2000; Agarwal and Mustafa, 2004; Lu and Vidal, 2006; Zhang et al., 2009), statistical methods (Tipping and Bishop, 1999,Sugaya and Kanatani, 2004, Gruber and Weiss, 2004,Yang et al., 2006, Ma et al., 2007,Rao et al., 2008, Rao et al., 2010), and spectral clustering-based methods (Boult and Brown, 1991; Yan and Pollefeys, 2006; Zhang et al., 2010; Goh and Vidal, 2007; Elhamifar and Vidal, 2009; Elhamifar and Vidal, 2010; Elhamifar and Vidal, 2013; Liu et al., 2010; Chen and Lerman, 2009). Among them, methods based on spectral clustering have been shown to perform very well for 
several applications in computer vision (see Vidal (2011) for a review and comparison of existing methods).

Spectral clustering-based methods (see von Luxburg, 2007 for a review) decompose the subspace clustering problem in two steps. In the first step, a symmetric affinity matrix $C=\left[c_{i j}\right]$ is constructed, where $c_{i j}=c_{j i} \geqslant 0$ measures whether points $i$ and $j$ belong to the same subspace. Ideally $c_{i j} \approx 1$ if points $i$ and $j$ are in the same subspace and $c_{i j} \approx 0$ otherwise. In the second step, a weighted undirected graph is constructed where the data points are the nodes and the affinities $c_{i j}$ are the weights. The segmentation of the data is then found by clustering the eigenvectors of the graph Laplacian using central clustering techniques, such as $k$-means. Arguably, the most difficult step is to build a good affinity matrix. This is because two points could be very close to each other, but lie in different subspaces (e.g., near the intersection of two subspaces). Conversely, two points could be far from each other, but lie in the same subspace.

Earlier methods for building an affinity matrix (Boult and Brown, 1991; Costeira and Kanade, 1998) compute the singular value decomposition (SVD) of the data matrix $D=U \Sigma V^{\top}$ and let $C=V_{1} V_{1}^{\top}$, where the columns of $V_{1}$ are the top $r=\operatorname{rank}(D)$ singular vectors of $D$. The rationale behind this choice is that $c_{i j}=0$ when points $i$ and $j$ are in different independent subspaces and the data are uncorrupted, as shown in Vidal et al. (2005). In practice, however, the data are often contaminated by noise and gross errors. In such cases, the equation $c_{i j}=0$ does not hold, even if the rank of the noiseless $D$ was given. Moreover, selecting a good value for $r$ becomes very difficult, because $D$ is full rank. Furthermore, the equation $c_{i j}=0$ is derived under the assumption that the subspaces are linear. In practice, many datasets are better modeled by affine subspaces.

More recent methods for building an affinity matrix address these issues by using techniques from sparse and low-rank representation. For instance, it is shown in Elhamifar and Vidal (2009, 2010,2013 ) that a point in a union of multiple subspaces admits a sparse representation with respect to the dictionary formed by all other data points, i.e., $D=D C$, where $C$ is sparse. It is also shown in Elhamifar and Vidal $(2009,2010,2013)$ that, if the subspaces are independent, the nonzero coefficients in the sparse representation of a point correspond to other points in the same subspace, i.e., if $c_{i j} \neq 0$, then points $i$ and $j$ belong to the same subspace. Moreover, the nonzero coefficients can be obtained by $\ell_{1}$ minimization. These coefficients are then converted into symmetric and nonnegative affinities, from which the segmentation is found using spectral clustering. A very similar approach is presented in Liu et al. (2010). The major difference is that a low-rank representation is used in lieu of the sparsest representation. While the same principle of representing a point as a linear combination of other points has been successfully used when the data are corrupted by noise and gross errors, from a theoretical viewpoint it is not clear that the above methods are effective when using a corrupted dictionary.

\subsection{Paper contributions}

In this paper, we propose a general optimization framework for solving the subspace clustering problem in the case of data corrupted by noise and/or gross errors. Given a corrupted data matrix $D \in \mathbb{R}^{M \times N}$, we wish to decompose it as the sum of a self-expressive, noise-free and outlier-free (clean) data matrix $A \in \mathbb{R}^{M \times N}$, a noise matrix $G \in \mathbb{R}^{M \times N}$, and a matrix of sparse gross errors $E \in \mathbb{R}^{M \times N}$. We assume that the columns of the matrix $A=\left[a_{1}, a_{2}, \ldots, a_{N}\right]$ are points in $\mathbb{R}^{M}$ drawn from a union of $n \geqslant 1$ low-dimensional linear subspaces of unknown dimensions $\left\{d_{i}\right\}_{i=1}^{n}$, where $d_{i} \ll M$. We also assume that $A$ is self-expressive, which means that the clean data points can be expressed as linear combinations of themselves, i.e.,
$a_{j}=\sum_{i=1}^{N} a_{i} c_{i j} \quad$ or $\quad A=A C$

where $C=\left[c_{i j}\right]$ is the matrix of coefficients. This constraint aims to capture the fact that a point in a linear subspace can be expressed as a linear combination of other points in the same subspace. Therefore, we expect $c_{i j}$ to be zero if points $i$ and $j$ are in different subspaces.

Notice that the constraint $A=A C$ is non-convex, because both $A$ and $C$ are unknown. This is an important difference with respect to existing methods, which enforce $D=D C$ where $D$ is the dictionary of corrupted data points. Another important difference is that we directly enforce $C$ to be symmetric, while existing methods symmetrize $C$ as a post-processing step.

The proposed framework, which we call Low Rank Subspace Clustering (LRSC), is based on solving the following non-convex optimization problem:

$\begin{array}{ll} & \min _{A, C, E, G}\|C\|_{*}+\frac{\tau}{2}\|A-A C\|_{F}^{2}+\frac{\alpha}{2}\|G\|_{F}^{2}+\gamma\|E\|_{1} \\ & \text { s.t. } D=A+G+E \text { and } C=C^{\top},\end{array}$

where $\|X\|_{*}=\sum_{i} \sigma_{i}(X),\|X\|_{F}^{2}=\sum_{i j} X_{i j}^{2}$ and $\|X\|_{1}=\sum_{i j}\left|X_{i j}\right|$ are, respectively, the nuclear, Frobenius and $\ell_{1}$ norms of $X$. The above formulation encourages:

- $C$ to be low-rank (by minimizing $\|C\|_{*}$ ),

- $A$ to be self-expressive (by minimizing $\|A-A C\|_{F}^{2}$ ),

- $G$ to be small (by minimizing $\|G\|_{F}^{2}$ ), and

- $E$ to be sparse (by minimizing $\|E\|_{1}$ ).

The main contribution of our work is to show that important particular cases of $P$ (see Table 1 ) can be solved in closed form from the SVD of the data matrix. In particular, we show that in the absence of gross errors (i.e., $\gamma=\infty$ ), $A$ and $C$ can be obtained by thresholding the singular values of $D$ and $A$, respectively. The thresholding is done using a novel polynomial thresholding operator, which reduces the amount of shrinkage with respect to existing methods. Indeed, when the self-similarity constraint $A=A C$ is enforced exactly (i.e., $\alpha=\infty$ ), the optimal solution for $A$ reduces to classical PCA, which does not perform any shrinkage. Moreover, the optimal solution for $C$ reduces to the affinity matrix for subspace clustering proposed by Costeira and Kanade (1998). In the case of data corrupted by gross errors, a closed-form solution appears elusive. We thus use an augmented Lagrange multipliers method. Each iteration of our method involves a polynomial thresholding of the singular values to reduce the rank and a regular shrinkage-thresholding to reduce gross errors.

Table 1

Particular cases of $P$ solved in this paper.

\begin{tabular}{ll}
\hline Relaxed & Exact \\
\hline Uncorrupted & \\
$P_{1}:$ Section 3.1 & $P_{2}$ : Section 3.2 \\
$0<\tau<\infty$ & $\tau=\infty$ \\
$\alpha=\infty$ & $\alpha=\infty$ \\
$\gamma=\infty$ & $\gamma=\infty$ \\
Noise & \\
$P_{3}:$ Section 4.1 & $P_{4}$ : Section 4.2 \\
$0<\tau<\infty$ & $\tau=\infty$ \\
$0<\alpha<\infty$ & $0<\alpha<\infty$ \\
$\gamma=\infty$ & $\gamma=\infty$ \\
Gross errors & \\
$P_{5}:$ Section 5.1 & $P_{6}:$ Section 5.2 \\
$0<\tau<\infty$ & $\tau=\infty$ \\
$0<\alpha<\infty$ & $0<\alpha<\infty$ \\
$0<\gamma<\infty$ & $0<\gamma<\infty$ \\
\hline
\end{tabular}




\subsection{Paper outline}

The remainder of the paper is organized as follows (see Table 1): Section 2 reviews existing results on sparse representation and rank minimization for subspace estimation and clustering as well as some background material needed for our derivations. Section 3 formulates the low rank subspace clustering problem for linear subspaces in the absence of noise or gross errors and derives a closed form solution for $A$ and $C$. Section 4 extends the results of Section 3 to data contaminated by noise and derives a closed form solution for $A$ and $C$ based on the polynomial thresholding operator. Section 5 extends the results to data contaminated by both noise and gross errors and shows that $A$ and $C$ can be found using alternating minimization. Section 6 presents experiments that evaluate our method on synthetic and real data. Section 7 gives the conclusions.

\section{Background}

In this section we review existing results on sparse representation and rank minimization for subspace estimation (Section 2.1) and and subspace clustering (Section 2.2).

\subsection{Subspace estimation by sparse representation and rank minimization}

\subsubsection{Low rank minimization}

Given a data matrix corrupted by Gaussian noise $D=A+G$, where $A$ is an unknown low-rank matrix and $G$ represents the noise, the problem of finding a low-rank approximation of $D$ can be formulated as

$\min _{A}\|D-A\|_{F}^{2}$ subject to $\operatorname{rank}(A) \leqslant r$.

The optimal solution to this (PCA) problem is given by $A=U \mathcal{H}_{\sigma_{r+1}}(\Sigma) V^{\top}$, where $D=U \Sigma V^{\top}$ is the SVD of $D, \sigma_{k}$ is the $k$ th singular value of $D$, and $\mathcal{H}_{\epsilon}(x)$ is the hard thresholding operator:

$\mathcal{H}_{\epsilon}(x)= \begin{cases}x & |x|>\epsilon \\ 0 & \text { else }\end{cases}$

When $r$ is unknown, the problem in (2) can be formulated as

$\min _{A} \operatorname{rank}(A)+\frac{\alpha}{2}\|D-A\|_{F}^{2}$,

where $\alpha>0$ is a parameter. Since the optimal solution of (2) for a fixed rank $r=\operatorname{rank}(A)$ is $A=U \mathcal{H}_{\sigma_{r+1}}(\Sigma) V^{\top}$, the problem in (4) is equivalent to

$\min _{r} r+\frac{\alpha}{2} \sum_{k>r} \sigma_{k}^{2}$

The optimal $r$ is the smallest $r$ such that $\sigma_{r+1} \leqslant \sqrt{2 / \alpha}$. Therefore, the optimal $A$ is given by $A=U \mathcal{H}_{\sqrt{\frac{2}{\alpha}}}(\Sigma) V^{\top}$.

Since rank minimization problems are in general NP hard, a common practice (see Recht et al., 2010) is to replace the rank of $A$ by its nuclear norm $\|A\|_{*}$, i.e., the sum of its singular values, which leads to the following convex problem

$\min _{A}\|A\|_{*}+\frac{\alpha}{2}\|D-A\|_{F}^{2}$,

where $\alpha>0$ is a user-defined parameter. It is shown in Cai et al. (2008) that the optimal solution to the problem in (6) is given by $A=U \mathcal{S}_{\frac{1}{\alpha}}(\Sigma) V^{\top}$, where $\mathcal{S}_{\epsilon}(x)$ is the shrinkage-thresholding operator

$\mathcal{S}_{\epsilon}(x)= \begin{cases}x-\epsilon & x>\epsilon \\ x+\epsilon & x<-\epsilon \\ 0 & \text { else. }\end{cases}$
Notice that the latter solution does not coincide with the one given by PCA, which performs hard-thresholding of the singular values of $D$ without shrinking them by $1 / \alpha$.

\subsubsection{Principal component pursuit}

While the above methods work well for data corrupted by Gaussian noise, they break down for data corrupted by gross errors. In Candès et al. (2011) this issue is addressed by assuming sparse gross errors, i.e., only a small percentage of the entries of $D$ are corrupted. Hence, the goal is to decompose the data matrix $D$ as the sum of a low-rank matrix $A$ and a sparse matrix $E$, i.e.,

$\min _{A, E} \operatorname{rank}(A)+\gamma\|E\|_{0} \quad$ s.t. $\quad D=A+E$

where $\gamma>0$ is a parameter and $\|E\|_{0}$ is the number of nonzero entries in $E$. Since this problem is in general NP hard, a common practice is to replace the rank of $A$ by its nuclear norm and the $\ell_{0}$ seminorm by the $\ell_{1}$ norm. It is shown in Candès et al. (2011) that, under broad conditions, the optimal solution to the problem in (8) is identical to that of the convex problem

$\min _{A, E}\|A\|_{*}+\gamma\|E\|_{1} \quad$ s.t. $\quad D=A+E$.

While a closed form solution to this problem is not known, convex optimization techniques can be used to find the minimizer. We refer the reader to Lin et al. (2011) for a review of numerous approaches. One such approach is the Augmented Lagrange Multiplier (ALM) method, an iterative approach for solving the following optimization problem

$\max _{Y} \min _{A, E}\|A\|_{*}+\gamma\|E\|_{1}+\langle Y, D-A-E\rangle+\frac{\alpha}{2}\|D-A-E\|_{F}^{2}$,

where the third term enforces the equality constraint via the matrix of Lagrange multipliers $Y$, while the fourth term (which is zero at the optimum) makes the cost function strictly convex. The optimization problem in (10) is solved using the inexact ALM method in (13). This method is motivated by the fact that the minimization over $A$ and $E$ for a fixed $Y$ can be re-written as

$\min _{A, E}\|A\|_{*}+\gamma\|E\|_{1}+\frac{\alpha}{2}\left\|D-A-E+\alpha^{-1} Y\right\|_{F}^{2}$.

Given $E$ and $Y$, it follows from the solution of (6) that the optimal $A$ is $A=U \mathcal{S}_{\alpha^{-1}}(\Sigma) V^{\top}$, where $U \Sigma V^{\top}$ is the SVD of $D-E+\alpha^{-1} Y$. Given $A$ and $Y$, the optimal $E$ satisfies

$-\alpha\left(D-A-E+\alpha^{-1} Y\right)+\gamma \operatorname{sign}(E)=0$.

It is shown in Lin et al. (2011) that this equation can be solved in closed form using the shrinkage-thresholding operator as $E=\mathcal{S}_{\gamma \alpha^{-1}}\left(D-A+\alpha^{-1} Y\right)$. Therefore, the inexact ALM method iterates the following steps till convergence

$(U, \Sigma, V)=\operatorname{svd}\left(D-E_{k}+\alpha_{k}^{-1} Y_{k}\right)$

$A_{k+1}=U \mathcal{S}_{\alpha_{k}^{-1}}(\Sigma) V^{\top}$

$E_{k+1}=\mathcal{S}_{\gamma \alpha_{k}^{-1}}\left(D-A_{k+1}+\alpha_{k}^{-1} Y_{k}\right)$

$Y_{k+1}=Y_{k}+\alpha_{k}\left(D-A_{k+1}-E_{k+1}\right)$

$\alpha_{k+1}=\rho \alpha_{k}$.

This ALM method is essentially an iterated thresholding algorithm, which alternates between thresholding the SVD of $D-E+Y / \alpha$ to get $A$ and thresholding $D-A+Y / \alpha$ to get $E$. The update for $Y$ is simply a gradient ascent step. Also, to guarantee the convergence of the algorithm, the parameter $\alpha$ is updated by choosing the parameter $\rho$ such that $\rho>1$ so as to generate a sequence $\alpha_{k}$ that goes to infinity. 


\subsection{Subspace clustering by sparse representation and rank minimization}

Consider now the more challenging problem of clustering data drawn from multiple subspaces. In what follows, we discuss two methods based on sparse and low-rank representation for addressing this problem.

\subsubsection{Sparse subspace clustering (SSC)}

The work of Elhamifar and Vidal (2009) shows that, in the case of uncorrupted data, an affinity matrix for solving the subspace clustering problem can be constructed by expressing each data point as a linear combination of all other data points. That is, we wish to find a matrix $C$ such that $D=D C$ and $\operatorname{diag}(C)=0$. In principle, this leads to an ill-posed problem with many possible solutions. To resolve this issue, the principle of sparsity is invoked. Specifically, every point is written as a sparse linear combination of all other data points by minimizing the number of nonzero coefficients. That is

$\min _{C} \sum_{i}\left\|C_{i}\right\|_{0}$ s.t. $D=D C$ and $\operatorname{diag}(C)=0$,

where $C_{i}$ is the $i$ th column of $C$. Since this problem is combinatorial, a simpler $\ell_{1}$ optimization problem is solved

$\min _{C}\|C\|_{1}$ s.t. $D=D C$ and $\operatorname{diag}(C)=0$.

It is shown in Elhamifar and Vidal $(2009,2010,2013)$ that, under some conditions on the subspaces and the data, the solutions to the optimization problems in (14) and (15) are such that $C_{i j}=0$ when points $i$ and $j$ are in different subspaces. In other words, the nonzero coefficients of the $i$ th column of $C$ correspond to points in the same subspace as point $i$. Therefore, one can use $C$ to define an affinity matrix as $|C|+\left|C^{\top}\right|$. The segmentation of the data is then obtained by applying spectral clustering (von Luxburg, 2007) to this affinity.

In the case of data contaminated by noise $G$, the SSC algorithm assumes that each data point can be written as a linear combination of other data points up to an error $G$, i.e., $D=D C+G$, and solves the following convex problem

$\min _{C, G}\|C\|_{1}+\frac{\alpha}{2}\|G\|_{F}^{2}$ s.t. $D=D C+G$ and $\operatorname{diag}(C)=0$.

In the case of data contaminated also by gross errors $E$, the SSC algorithm assumes that $D=D C+G+E$, where $E$ is sparse. Since both $C$ and $E$ are sparse, the equation $D=D C+G+E=$ $[D I]\left[C^{\top} E^{\top}\right]^{\top}+G$ means that each point is written as a sparse linear combination of a dictionary composed of all other data points plus the columns of the identity matrix $I$. Thus, one can find $C$ by solving the following convex optimization problem

$\min _{C, G, E}\|C\|_{1}+\frac{\alpha}{2}\|G\|_{F}^{2}+\gamma\|E\|_{1}$

s.t. $D=D C+G+E$ and $\operatorname{diag}(C)=0$.

While SSC works well in practice, until recently there was no theoretical guarantee that, in the case of corrupted data, the nonzero coefficients correspond to points in the same subspace. We refer the reader to Soltanolkotabi et al. (2013) for very recent results in this direction. Moreover, notice that the model is not really a subspace plus error model, because a contaminated data point is written as a linear combination of other contaminated points plus an error. To the best of our knowledge, there is no method that tries to simultaneously recover a clean dictionary and cluster the data within this framework.

\subsubsection{Low rank representation (LRR)}

This algorithm (Liu et al., 2010) is very similar to SSC, except that it aims to find a low-rank representation instead of a sparse representation. LRR is motivated by the fact that for uncorrupted data drawn from $n$ independent subspaces of dimensions $\left\{d_{i}\right\}_{i=1}^{n}$, the data matrix $D$ is low rank because $r=\operatorname{rank}(D)=\sum_{i=1}^{n} d_{i}$, which is assumed to be much smaller than $\min \{M, N\}$. Therefore, the equation $D=D C$ has a solution $C$ that is low-rank. The LRR algorithm finds $C$ by solving the following convex optimization problem

$\min _{C}\|C\|_{*}$ s.t. $D=D C$.

It is shown in Liu et al. (2011) that the optimal solution to (18) is given by $C=V_{1} V_{1}^{\top}$, where $D=U_{1} \Sigma_{1} V_{1}^{\top}$ is the rank $r$ SVD of $D$. Notice that this solution for $C$ coincides with the affinity matrix proposed by Costeira and Kanade (1998), as described in the introduction. It is shown in Vidal et al. (2008) that this matrix is such that $C_{i j}=0$ when points $i$ and $j$ are in different subspaces, hence it can be used to build an affinity matrix.

In the case of data contaminated by noise or gross errors, the LRR algorithm solves the convex optimization problem

$\min _{C}\|C\|_{*}+\gamma\|E\|_{2,1}$ s.t. $D=D C+E$,

where $\|E\|_{2,1}=\sum_{k=1}^{N} \sqrt{\sum_{j=1}^{N}\left|E_{j k}\right|^{2}}$ is the $\ell_{2,1}$ norm of the matrix of errors $E$. Notice that the problem in (19) is analogous to that in (17), except that the $\ell_{1}$ norms of $C$ and $E$ are replaced by the nuclear norm of $C$ and the $\ell_{2,1}$ norm of $E$, respectively. The nuclear norm is a convex relaxation of the rank of $C$, while the $\ell_{2,1}$ norm is a convex relaxation of the number of nonzero columns of $E$. Therefore, the LRR algorithm aims to minimize the rank of the matrix of coefficients and the number of corrupted data points, while the SSC algorithm aims to minimize the number of nonzero coefficients and the number of corrupted entries. As before, the optimal $C$ is used to define an affinity matrix $|C|+\mid C^{\top}$ and the segmentation of the data is obtained by applying spectral clustering to this affinity.

\section{Low rank subspace clustering with uncorrupted data}

In this section, we consider the low rank subspace clustering problem $P$ in the case of uncorrupted data, i.e., $\alpha=\infty$ and $\gamma=\infty$ so that $G=E=0$ and $D=A$. In Section 3.1, we show that the optimal solution for $C$ can be obtained in closed form from the SVD of $A$ by applying a nonlinear thresholding to its singular values. In Section 3.2, we assume that the self-expressiveness constraint is satisfied exactly, i.e., $\tau=\infty$ so that $A=A C$. As shown in Liu et al. (2011), the optimal solution to this problem can be obtained by hard thresholding the singular values of $A$. Here, we provide a simpler derivation of this result.

\subsection{Uncorrupted data and relaxed constraints}

Consider the optimization problem $P$ with uncorrupted data and relaxed self-expressiveness constraint, i.e., $\tau<\infty$ so that $A \approx A C$. In this case, the problem $P$ reduces to

$\left(P_{1}\right) \quad \min _{C}\|C\|_{*}+\frac{\tau}{2}\|A-A C\|_{F}^{2}$ s.t. $C=C^{\top}$.

Notice that $P_{1}$ is convex on $C$, but not strictly convex. Therefore, we do not know a priori if the minimizer of $P_{1}$ is unique. The following theorem shows that the minimizer of $P_{1}$ is unique and can be computed in closed form from the SVD of $A$.

Theorem 1. Let $A=U \Lambda V^{\top}$ be the SVD of $A$, where the diagonal entries of $\Lambda=\operatorname{diag}\left(\left\{\lambda_{i}\right\}\right)$ are the singular values of $A$ in decreasing order. The optimal solution to $P_{1}$ is given by 
$C=V \mathcal{P}_{\tau}(\Lambda) V^{\top}=V_{1}\left(I-\frac{1}{\tau} \Lambda_{1}^{-2}\right) V_{1}^{\top}$

where the operator $\mathcal{P}_{\tau}$ acts on the diagonal entries of $\Lambda$ as

$\mathcal{P}_{\tau}(x) \doteq\left\{\begin{array}{ll}1-\frac{1}{\tau x^{2}} & x>1 / \sqrt{\tau} \\ 0 & x \leqslant 1 / \sqrt{\tau}\end{array}\right.$,

and $U=\left[U_{1} U_{2}\right], \Lambda=\operatorname{diag}\left(\Lambda_{1}, \Lambda_{2}\right)$ and $V=\left[V_{1} V_{2}\right]$ are partitioned according to the sets $\mathbf{I}_{1}=\left\{i: \lambda_{i}>1 / \sqrt{\tau}\right\}$ and $\mathbf{I}_{2}=\left\{i: \lambda_{i} \leqslant 1 / \sqrt{\tau}\right\}$. Moreover, the optimal value of $P_{1}$ is

$\Phi_{\tau}(A) \doteq \sum_{i \in \mathbf{I}_{1}}\left(1-\frac{1}{2 \tau} \lambda_{i}^{-2}\right)+\frac{\tau}{2} \sum_{i \in \mathbf{I}_{2}} \lambda_{i}^{2}$

\section{Proof. See Appendix B.}

Notice that the optimal solution for $C$ is obtained by applying a nonlinear thresholding $\mathcal{P}_{\tau}$ to the singular values of $A$. Singular values smaller than $\frac{1}{\sqrt{\tau}}$ are mapped to zero, while larger singular values are mapped closer to one. Notice also that the optimal value of $P_{1}, \Phi_{\tau}(A)$, is a decomposable function of the singular values of $A$, as are the Frobenius and nuclear norms of $A,\|A\|_{F}^{2}=\sum \lambda_{i}^{2}$ and $\|A\|_{*}=\sum \lambda_{i}$, respectively. However, unlike $\|A\|_{F}$ or $\|A\|_{*}, \Phi_{\tau}(A)$ is not a convex function of $A$ because $\Phi_{\tau}(\lambda)$ is quadratic near zero and saturates as $\lambda$ increases, as illustrated in Fig. 1(a). Interestingly, as $\tau$ goes to infinity, $\Phi_{\tau}(A)$ approaches $\operatorname{rank}(A)$, as we shall see. Therefore, we may view $\Phi_{\tau}(A)$ as a non-convex relaxation of $\operatorname{rank}(A)$.

\subsection{Uncorrupted data and exact constraints}

Consider now the optimization problem $P$ with uncorrupted data and exact self-expressiveness constraint, i.e., $\tau=\infty$ so that $A \approx A C$. This leads to the following optimization problem

$\left(P_{2}\right) \quad \min _{C}\|C\|_{*}$ s.t. $A=A C$ and $C=C^{\top}$.

The following Theorem shows that the Costeira and Kanade affinity matrix $C=V_{1} V_{1}^{\top}$ is the optimal solution to $P_{2}$. The theorem follows as a corollary of Theorem 1 by letting $\tau \rightarrow \infty$. An alternative proof can be found in Liu et al. (2011). Here, we provide a simpler and more direct proof.

Theorem 2. Let $A=U \Lambda V^{\top}$ be the SVD of $A$, where the diagonal entries of $\Lambda=\operatorname{diag}\left(\left\{\lambda_{i}\right\}\right)$ are the singular values of $A$ in decreasing order. The optimal solution to $P_{2}$ is given by

$C=V_{1} V_{1}^{\top}$,

where $V=\left[V_{1} V_{2}\right]$ is partitioned according to the sets $\mathbf{I}_{1}=\left\{i: \lambda_{i}>0\right\}$ and $\mathbf{I}_{2}=\left\{i: \lambda_{i}=0\right\}$. Moreover, the optimal value is

$\Phi_{\infty}(A)=\sum_{i \in \mathbf{I}_{1}} 1=\operatorname{rank}(A)$.

Proof. Let $C=U_{C} \Delta U_{C}^{\top}$ be the eigenvalue decomposition (EVD) of $C$. Then $A=A C$ can be rewritten as $U \Lambda V^{\top}=U \Lambda V^{\top} U_{C} \Delta U_{C}^{\top}$, which reduces to

$\Lambda V^{\top} U_{C}=\Lambda V^{\top} U_{C} \Delta$

since $U^{\top} U=I$ and $U_{C}^{\top} U_{C}=I$. Let $W=V^{\top} U_{C}=\left[w_{1}, \ldots, w_{N}\right]$. Then, $\Lambda w_{j}=\Lambda w_{j} \delta_{j}$ for all $j=1, \ldots, N$. This means that $\delta_{j}=1$ if $\Lambda w_{j} \neq \mathbf{0}$ and $\delta_{j}$ is arbitrary otherwise. Since our goal is to minimize $\|C\|_{*}=\|\Delta\|_{*}=\sum_{j=1}^{N}\left|\delta_{j}\right|$, we need to set as many $\delta_{j}$ to zero as possible. Since $A=A C$ implies that $\operatorname{rank}(A) \leqslant \operatorname{rank}(C)$, we can set at most $N-\operatorname{rank}(A)$ of the $\delta_{j}$ to zero and the remaining $\operatorname{rank}(A)$ of the $\delta_{j}$ must be equal to one. Now, if $\delta_{j}=0$, then $\Lambda w_{j}=\Lambda_{1} V_{1}^{\top} U_{C} e_{j}=\mathbf{0}$, where $e_{j}$ is the $j$ th column of the identity. This means that the columns of $U_{C}$ associated to $\delta_{j}=0$ must be orthogonal to the columns of $V_{1}$, and hence the columns of $U_{C}$ associated with $\delta_{j}=1$ must be in the range of $V_{1}$. Thus, $U_{C}=\left[\begin{array}{ll}V_{1} R_{1} & U_{2} R_{2}\end{array}\right] \Pi$ for some rotation matrices $R_{1}$ and $R_{2}$, and permutation matrix $\Pi$, and so the optimal $C$ is

$C=\left[\begin{array}{ll}V_{1} R_{1} & V_{2} R_{2}\end{array}\right]\left[\begin{array}{ll}I & \mathbf{0} \\ \mathbf{0} & \mathbf{0}\end{array}\right]\left[\begin{array}{ll}V_{1} R_{1} & V_{2} R_{2}\end{array}\right]^{\top}=V_{1} V_{1}^{\top}$,

as claimed.

\section{Low rank subspace clustering with noisy data}

In this section, we consider the low rank subspace clustering problem $P$ in the case of noisy data, i.e., $\lambda=\infty$, so that $E=0$ and $D=A+G$. While in principle the resulting problem appears to be very similar to those in eqs. (16) and (19), there are a number of differences. First, notice that instead of expressing the noisy data as a linear combination of itself plus noise, i.e., $D=D C+G$, we search for a clean dictionary, $A$, which is self-expressive, i.e., $A=A C$. We then assume that the data are obtained by adding noise to the clean dictionary, i.e., $D=A+G$. As a consequence, our method searches simultaneously for a clean dictionary $A$, the coefficients $C$ and the noise $G$. Second, the main difference with (16) is that the $\ell_{1}$ norm of the matrix of the coefficients is replaced by the nuclear norm. Third, the main difference with (19) is that the $\ell_{2,1}$ norm of the matrix of the noise is replaced by the Frobenius norm. Fourth, our method enforces the symmetry of the affinity matrix as part of the optimization problem, rather than as a post-processing step.

As we will show in this section, these modifications result in a key difference between our method and the state of the art: while the solution to (16) requires $\ell_{1}$ minimization and the solution to (19) requires an ALM method, the solution to $P$ with noisy data can be computed in closed form from the SVD of the data matrix $D$. For the relaxed problem, $P_{3}$, the closed-form solution for $A$ is found by applying a polynomial thresholding to the singular values of $D$, as we will see in Section 4.1. For the exact problem, $P_{4}$, the closed-form solution for $A$ is given by classical PCA, except that the number of principal components can be automatically determined, as we will see in Section 4.2.

\subsection{Noisy data and relaxed constraints}

Consider the optimization problem $P$ with noisy data and relaxed self-expressiveness constraint, i.e.

$\left(P_{3}\right) \quad \min _{A, C}\|C\|_{*}+\frac{\tau}{2}\|A-A C\|_{F}^{2}+\frac{\alpha}{2}\|D-A\|_{F}^{2}$ s.t. $C=C^{\top}$.

The key difference between $P_{3}$ and the problem $P_{1}$ considered in Section 3.1 is that $A$ is now unknown. Hence the cost function in $P_{3}$ is not convex in $(A, C)$ because of the product $A C$. Nonetheless, we will show in this subsection that the optimal solution is still unique, unless one of the singular values of $D$ satisfies a constraint that depends on $\alpha$ and $\tau$. In such a degenerate case, the problem has two optimal solutions. Moreover, the optimal solutions for both $A$ and $C$ can be computed in closed form from the SVD of $D$, as stated in the following theorem.

Theorem 3. Let $D=U \Sigma V^{\top}$ be the SVD of the data matrix $D$. The optimal solutions to $P_{3}$ are of the form

$A=U \Lambda V^{\top}$ and $C=V \mathcal{P}_{\tau}(\Lambda) V^{\top}$,

where each entry of $\Lambda=\operatorname{diag}\left(\lambda_{1}, \ldots, \lambda_{n}\right)$ is obtained from each entry of $\Sigma=\operatorname{diag}\left(\sigma_{1}, \ldots, \sigma_{n}\right)$ as the solutions to 


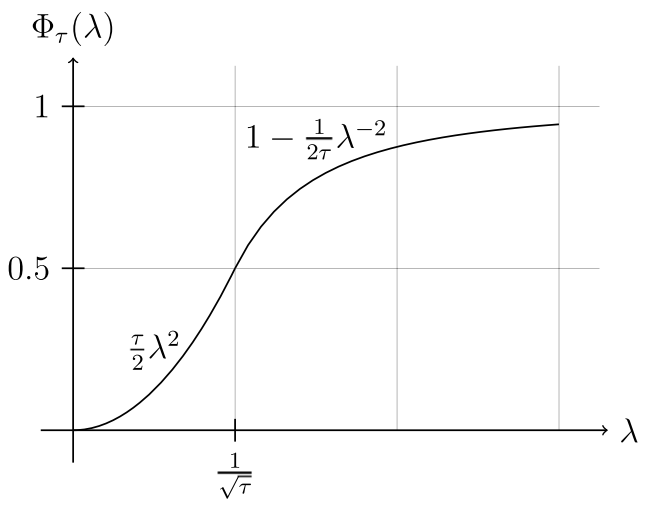

(a) Plot of $\Phi_{\tau}(\lambda)$.

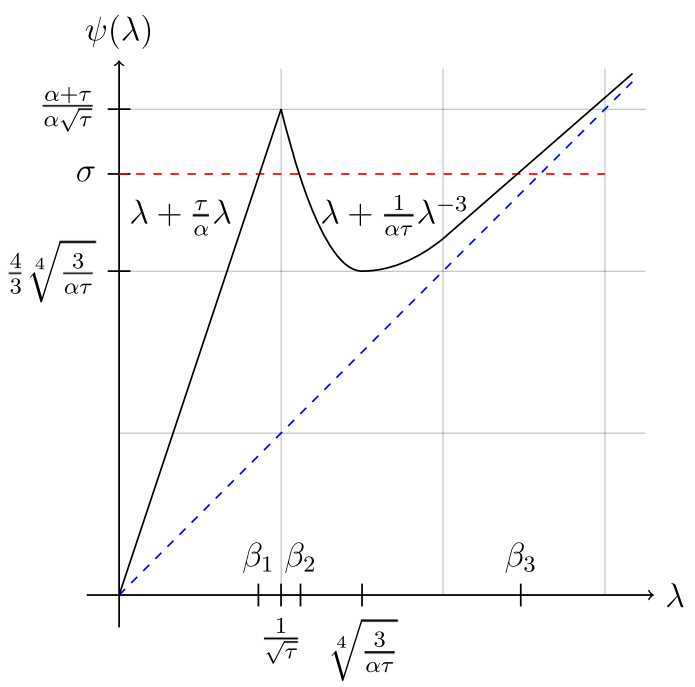

(c) Plot of $\psi(\lambda)$ when $3 \tau>\alpha$.

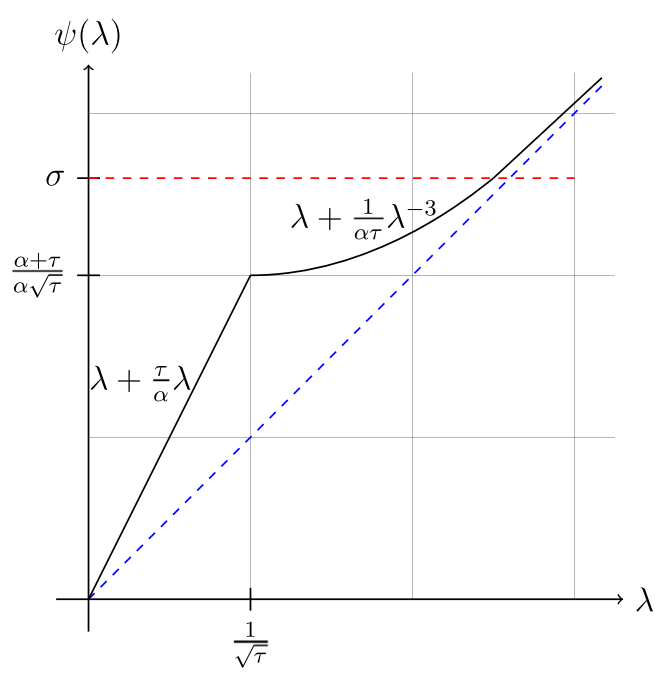

(b) Plot of $\psi(\lambda)$ when $3 \tau \leq \alpha$.

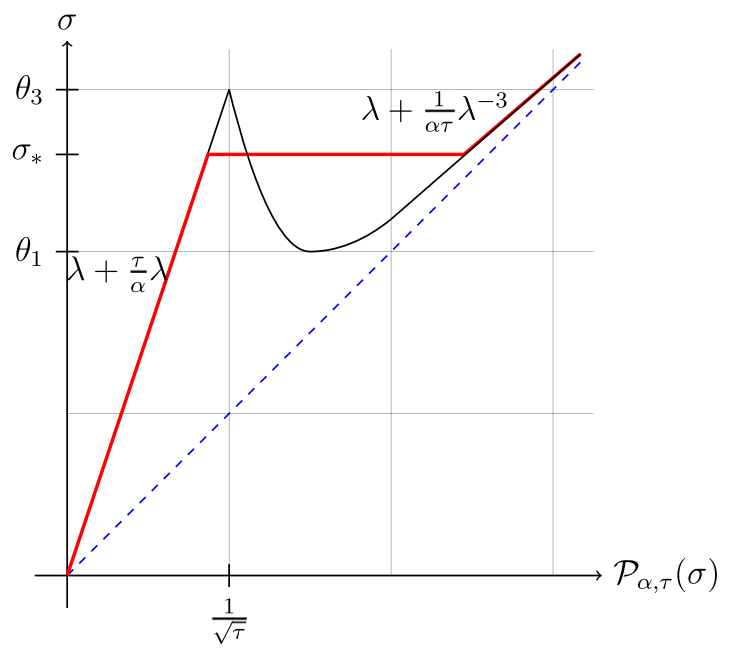

(d) The polynomial thresholding operator.

Fig. 1. Plots of $\Phi_{\tau}, \psi$ and $\mathcal{P}_{\alpha, \tau}$.

$\sigma=\psi(\lambda) \doteq \begin{cases}\lambda+\frac{1}{\alpha \tau} \lambda^{-3} & \text { if } \lambda>1 / \sqrt{\tau}, \\ \lambda+\frac{\tau}{\alpha} \lambda & \text { if } \lambda \leqslant 1 / \sqrt{\tau},\end{cases}$

that minimize

$\phi(\lambda, \sigma) \doteq \frac{\alpha}{2}(\sigma-\lambda)^{2}+ \begin{cases}1-\frac{1}{2 \tau} \lambda^{-2} & \lambda>1 / \sqrt{\tau}, \\ \frac{\tau}{2} \lambda^{2} & \lambda \leqslant 1 / \sqrt{\tau} .\end{cases}$

Proof. Notice that when $A$ is fixed, $P_{3}$ reduces to $P_{1}$. Therefore, it follows from Theorem 1 that, given $A$, the optimal solution for $C$ is $C=V \mathcal{P}_{\tau}(\Lambda) V^{\top}$, where $A=U \Lambda V^{\top}$ is the SVD of $A$. Moreover, it follows from (22) that if we replace the optimal $C$ into the cost of $P_{3}$, then $P_{3}$ is reduces to

$\min _{A} \Phi_{\tau}(A)+\frac{\alpha}{2}\|D-A\|_{F}^{2}$.

It is easy to see that, because $\Phi_{\tau}(A)$ is a decomposable function of the singular values of $A$, the optimal solution for $A$ in (30) must be of the form $A=U \Lambda V^{\top}$ for some $\Lambda=\operatorname{diag}\left(\left\{\lambda_{i}\right\}_{i=1}^{n}\right)$. After substituting this expression for $A$ into (30), we obtain that $\Lambda$ must minimize $\Phi_{\tau}(\Lambda)+\frac{\alpha}{2}\|\Sigma-\Lambda\|_{F}^{2}=\sum_{i=1}^{n} \phi\left(\lambda_{i}, \sigma_{i}\right)$. Therefore, each $\lambda_{i}$ can be obtained independently as the minimizer of $\phi\left(\lambda, \sigma_{i}\right)$. After taking the derivative of $\phi(\lambda, \sigma)$ w.r.t. $\lambda$ and setting it to zero, we obtain $\sigma=\psi(\lambda)$. Therefore, $\lambda_{i}$ is the solution of $\sigma_{i}=\psi(\lambda)$ that minimizes $\phi\left(\lambda, \sigma_{i}\right)$, as claimed. For a more detailed proof, we refer the reader to Appendix $B$.

It follows from Theorem 3 that the singular values of $A$ can be obtained from those of $D$ by solving the equation $\sigma=\psi(\lambda)$. When $3 \tau \leqslant \alpha$, the solution for $\lambda$ is unique (see Fig. 1(b)). However, when $3 \tau>\alpha$ there are three possible solutions (see Fig. 1(c)). The following theorem shows that, in general, only one of these solutions is the global minimizer. Moreover, this solution can be obtained by applying a polynomial thresholding operator $\lambda=\mathcal{P}_{\alpha, \tau}(\sigma)$ to the singular values of $D$.

Theorem 4. There exists a $\sigma_{*}>0$ such that the solutions to (28) that minimize (29) can be computed as

$\lambda=\mathcal{P}_{\alpha, \tau}(\sigma) \doteq \begin{cases}\beta_{1}(\sigma) & \text { if } \sigma \leqslant \sigma_{*} \\ \beta_{3}(\sigma) & \text { if } \sigma>\sigma_{*},\end{cases}$

where $\beta_{1}(\sigma) \doteq \frac{\alpha}{\alpha+\tau} \sigma$ and $\beta_{3}(\sigma)$ is the real root of the polynomial

$p(\lambda)=\lambda^{4}-\sigma \lambda^{3}+\frac{1}{\alpha \tau}=0$ 
that minimizes (29). If $3 \tau \leqslant \alpha$, the solution for $\lambda$ is unique and

$\sigma_{*}=\psi\left(\frac{1}{\sqrt{\tau}}\right)=\frac{\alpha+\tau}{\alpha \sqrt{\tau}}$

If $3 \tau>\alpha$, the solution for $\lambda$ is unique, except when $\sigma$ satisfies

$\phi\left(\beta_{1}(\sigma), \sigma\right)=\phi\left(\beta_{3}(\sigma), \sigma\right)$,

and $\sigma_{*}$ must lie in the range

$\frac{4}{3} \sqrt[4]{\frac{3}{\alpha \tau}}<\sigma_{*}<\frac{\alpha+\tau}{\alpha \sqrt{\tau}}$

\section{Proof. See Appendix B.}

It follows from Theorems 3 and 4 that the optimal solutions to $P_{3}$ can be obtained from the SVD of $D=U \Sigma V^{\top}$ as

$A=U \mathcal{P}_{\alpha, \tau}(\Sigma) V^{\top} \quad$ and $\quad C=V \mathcal{P}_{\tau}\left(\mathcal{P}_{\alpha, \tau}(\Sigma)\right) V^{\top}$.

However, to compute $\mathcal{P}_{\alpha, \tau}$, we need to compute the threshold $\sigma^{*}$. Unfortunately, finding a formula for $\sigma_{*}$ is not straightforward, because it requires solving (34) (see B for details). While this equation can be solved numerically for each $\alpha$ and $\tau$, a simple closed-form formula can be obtained when $\frac{1}{\alpha \tau} \simeq 0$ (relative to $\sigma$ ). In this case, the quartic becomes $p(\lambda)=\lambda^{4}-\sigma \lambda^{3}=0$, which can be immediately solved and yields three solutions that are equal to 0 and are hence out of the range $\lambda>1 / \sqrt{\tau}$. The only valid solution to the quartic is

$\lambda=\sigma \quad \forall \sigma: \sigma>1 / \sqrt{\tau}$.

Thus, a simpler thresholding procedure can be obtained by approximating the thresholding function with two piecewise linear functions. One is exact (when $\lambda \leqslant 1 / \sqrt{\tau}$ ) and the other one is approximate (when $\lambda>1 / \sqrt{\tau}$ ). The approximation, however, is quite accurate for a wide range of values for $\alpha$ and $\tau$. Since we have two linear functions, we can easily find a threshold for $\sigma$ as the value $\widetilde{\sigma}_{*}$ at which the discontinuity happens. To do so, we can plug in the given solutions in (34). We obtain

$\frac{\alpha \tau}{2(\alpha+\tau)} \tilde{\sigma}_{*}^{2}=1-\frac{1}{2 \tau \widetilde{\sigma}_{*}^{2}}$.

This gives 4 solutions, out of which the only suitable one is

$\tilde{\sigma}_{*}=\sqrt{\frac{\alpha+\tau}{\alpha \tau}+\sqrt{\frac{\alpha+\tau}{\alpha^{2} \tau}}}$.

Finally, the approximate polynomial thresholding operator can be written as

$\lambda=\widetilde{\mathcal{P}}_{\alpha, \tau}(\sigma)= \begin{cases}\sigma & \text { if } \sigma>\widetilde{\sigma}_{*} \\ \frac{\alpha}{\alpha+\tau} \sigma & \text { if } \sigma \leqslant \widetilde{\sigma}_{*} .\end{cases}$

Notice that as $\tau$ increases, the largest singular values of $D$ are preserved, rather than shrunk by the operator $\mathcal{S}_{\alpha^{-1}}$ in (7). Notice also that the smallest singular values of $D$ are shrunk by scaling them down, as opposed to subtracting a threshold.

\subsection{Noisy data and exact constraints}

Assume now that the data are generated from the exact selfexpressive model, $A=A C$, and contaminated by noise, i.e., $D=A+G$. This leads to the optimization problem

$\left(P_{4}\right) \quad \min _{A, C}\|C\|_{*}+\frac{\alpha}{2}\|D-A\|_{F}^{2}$ s.t. $A=A C$ and $C=C^{\top}$.

This problem can be seen as the limiting case of $P_{3}$ with $\tau \rightarrow \infty$. In this case, $\beta_{1}(\sigma)=\sigma, \beta_{3}(\sigma)=0$ and $\sigma_{*}=\sqrt{\frac{2}{\alpha}}$, hence the polynomial thresholding operator $\mathcal{P}_{\alpha, \tau}$ in (31) reduces to the hard thresholding operator $\mathcal{H}_{\sqrt{\frac{2}{\alpha}}}$ in (3). Therefore, the optimal $A$ can be obtained from the SVD of $D=U \Sigma V^{\top}$ as $A=U \mathcal{H}_{\sqrt{\frac{2}{\alpha}}}(\Sigma) V^{\top}$, while the optimal $C$ is given by Theorem 2 .

Theorem 5. Let $D=U \Sigma V^{\top}$ be the SVD of the data matrix $D$. The optimal solution to $P_{4}$ is given by

$A=U_{1} \Sigma_{1} V_{1}^{\top}$ and $C=V_{1} V_{1}^{\top}$,

where $\Sigma_{1}$ contains the singular values of $D$ that are larger than $\sqrt{\frac{2}{\alpha^{\prime}}}$, and $U_{1}$ and $V_{1}$ contain the corresponding singular vectors.

\section{Low rank subspace clustering with corrupted data}

In this section, we consider the low-rank subspace clustering problem in the case of data corrupted by both noise and gross errors, i.e., problem $P$. Similar to the case of noisy data discussed in Section 4, the major difference between $P$ and the problems in (17) and (19) is that, rather than using a corrupted dictionary, we search simultaneously for the clean dictionary $A$, the low-rank coefficients $C$ and the sparse errors $E$. Also, notice that the $\ell_{1}$ norm of the matrix of coefficients is replaced by the nuclear norm, the $\ell_{2,1}$ norm of the matrix of errors is replaced by the $\ell_{1}$ norm, and we enforce the symmetry of the affinity matrix as part of the optimization problem rather than as a post-processing. A closed form solution to the low-rank subspace clustering problem in the case of data corrupted by noise and gross errors appears elusive at this point. Therefore, we propose to solve $P$ using an alternating minimization approach.

\subsection{Corrupted data and relaxed constraints}

\subsubsection{Iterative polynomial thresholding (IPT)}

We begin by considering the relaxed problem $P$, which is equivalent to

$$
\begin{aligned}
& \left(P_{5}\right) \quad \min _{A, C, E}\|C\|_{*}+\frac{\tau}{2}\|A-A C\|_{F}^{2}+\frac{\alpha}{2}\|D-A-E\|_{F}^{2}+\gamma\|E\|_{1} \\
& \text { s.t. } C=C^{\top} .
\end{aligned}
$$

When $E$ is fixed, this problem reduces to $P_{3}$, except that $D$ is replaced by $D-E$. Therefore, it follows from (36) that $A$ and $C$ can be computed from the SVD of $D-E=U \Sigma V^{\top}$ as

$A=U \mathcal{P}_{\alpha, \tau}(\Sigma) V^{\top}$ and $\quad C=V \mathcal{P}_{\tau}\left(\mathcal{P}_{\alpha, \tau}(\Sigma)\right) V^{\top}$,

where $\mathcal{P}_{\tau}$ is the operator in (21) and $\mathcal{P}_{\alpha, \tau}$ is the polynomial thresholding operator in (31). When $A$ and $C$ are fixed, the optimal solution for $E$ satisfies

$-\alpha(D-A-E)+\gamma \operatorname{sign}(E)=0$.

This equation can be solved in closed form by using the shrinkage-thresholding operator in (7) and the solution is

$E=\mathcal{S}_{\frac{\gamma}{\alpha}}(D-A)$.

This suggest an iterative thresholding algorithm that, starting from $A_{0}=D$ and $E_{0}=0$, alternates between applying polynomial thresholding to $D-E_{k}$ to obtain $A_{k+1}$ and applying shrinkage-thresholding to $D-A_{k+1}$ to obtain $E_{k+1}$, i.e.,

$\left(U_{k}, \Sigma_{k}, V_{k}\right)=\operatorname{svd}\left(D-E_{k}\right)$

$A_{k+1}=U_{k} \mathcal{P}_{\alpha, \tau}\left(\Sigma_{k}\right) V_{k}^{\top}$

$E_{k+1}=\mathcal{S}_{\gamma \alpha^{-1}}\left(D-A_{k+1}\right)$.

Notice that we do not need to compute $C$ at each iteration because the updates for $A$ and $E$ do not depend on $C$. Therefore, we can obtain $C$ from $A$ upon convergence. Although the optimization 
problem in (42) is non-convex, the algorithm in (45) is guaranteed to converge, as shown in Tseng (2001). Specifically, it follows from Theorem 1 that the optimization problem in (42) is equivalent to the minimization of the cost function

$f(A, E)=\Phi_{\tau}(A)+\frac{\alpha}{2}\|D-A-E\|_{F}^{2}+\gamma\|E\|_{1}$.

It is easy to see that the algorithm in (45) is a coordinate descent method applied to the minimization of $f$. This function is continuous, has a compact level set $\left\{(A, E): f(A, E) \leqslant f\left(A_{0}, E_{0}\right)\right\}$, and has at most one minimum in $E$ as per (44). Therefore, it follows from Theorem 4.1 part (c) in Tseng (2001) that the algorithm in (45) converges to a coordinate-wise minimum of $f$.

Notice, however, that this minimum is not guaranteed to be a global minimum. Moreover, in practice its convergence can be slow as observed in Lin et al. (2011) for similar problems.

\subsubsection{Alternating direction method of multipliers (ADMM)}

We now propose an alternative solution to $P_{5}$ in which we enforce the constraint $D=A+E$ exactly. This means that we tolerate outliers, but we do not tolerate noise. Using the method of multipliers, this problem can be formulated as

$$
\begin{aligned}
& \max _{Y} \min _{A, E, C: C=C^{\top}}\|C\|_{*}+\frac{\tau}{2}\|A-A C\|_{F}^{2}+\frac{\mu}{2}\|D-A-E\|_{F}^{2} \\
& \quad+\langle Y, D-A-E\rangle+\gamma\|E\|_{1} .
\end{aligned}
$$

In this formulation, the term with $\mu$ does not play the role of penalizing the noise $G=D-A-E$, as before. Instead, it augments the Lagrangian with the squared norm of the constraint.

To solve the minimization problem over $(A, E, C)$, notice that when $E$ is fixed the optimization over $A$ and $C$ is equivalent to

$\min _{A, C}\|C\|_{*}+\frac{\tau}{2}\|A-A C\|_{F}^{2}+\frac{\mu}{2}\left\|D-A-E+\mu^{-1} Y\right\|_{F}^{2} \quad$ s.t. $C=C^{\top}$.

It follows from (36) that the optimal solutions for $A$ and $C$ can be computed from the SVD of $D-E+\mu^{-1} Y=U \Sigma V^{\top}$ as

$A=U \mathcal{P}_{\mu, \tau}(\Sigma) V^{\top} \quad$ and $\quad C=V \mathcal{P}_{\tau}\left(\mathcal{P}_{\mu, \tau}(\Sigma)\right) V^{\top}$,

Conversely, when $A$ and $C$ are fixed, the optimization problem over $E$ reduces to

$\min _{E} \frac{\mu}{2}\left\|D-A-E+\mu^{-1} Y\right\|_{F}^{2}+\gamma\|E\|_{1}$.

As discussed in Section 2.1, the optimal solution for $E$ is given as $E=\mathcal{S}_{\gamma / \mu}\left(D-A+\mu^{-1} Y\right)$.

Given $A$ and $E$, the ADMM algorithm updates $Y$ using gradient ascent with step size $\mu$, which gives $Y \leftarrow Y+\mu(D-A-E)$. Therefore, starting from $A_{0}=D, E_{0}=0$ and $Y_{0}=0$, we obtain the following ADMM for solving the low-rank subspace clustering problem in the presence of gross corruptions,

$\left(U_{k}, \Sigma_{k}, V_{k}\right)=\operatorname{svd}\left(D-E_{k}+\mu_{k}^{-1} Y_{k}\right)$

$A_{k+1}=U_{k} \mathcal{P}_{\mu_{k}, \tau}\left(\Sigma_{k}\right) V_{k}^{\top}$

$E_{k+1}=\mathcal{S}_{\gamma \mu_{k}^{-1}}\left(D-A_{k+1}+\mu_{k}^{-1} Y_{k}\right)$

$Y_{k+1}=Y_{k}+\mu_{k}\left(D-A_{k+1}-E_{k+1}\right)$

$\mu_{k+1}=\rho \mu_{k}$,

where $\rho>1$ is a parameter. As in the case of the IPT method, $C$ is obtained from $A$ upon convergence. Experimentally, we have observed that our method always converges. However, while the convergence of the ADMM is well studied for convex problems, we are not aware of any extensions to the nonconvex case.

\subsection{Corrupted data and exact constraints}

Let us now consider the low rank subspace clustering problem $P$, where the constraint $A=A C$ is enforced exactly, i.e.,

$$
\begin{aligned}
& \min _{A, C, E, G}\|C\|_{*}+\frac{\alpha}{2}\|G\|_{F}^{2}+\gamma\|E\|_{1} \\
& \text { s.t. } D=A+G+E, A=A C \text { and } C=C^{\top} .
\end{aligned}
$$

This problem can be seen as the limiting case of $P_{5}$ with $\tau \rightarrow \infty$. In this case, the polynomial thresholding operator $\mathcal{P}_{\alpha, \tau}$ becomes the hard thresholding operator $\mathcal{H}_{\sqrt{\frac{2}{\alpha}}}$. Therefore, we can solve $P_{6}$ using the IPT and ADMM algorithms described in Section 5.1 with $\mathcal{P}_{\alpha, \tau}$ replaced by $\mathcal{H}_{\sqrt{\frac{2}{\alpha}}}$.

\section{Experiments}

In this section we evaluate the performance of LRSC on two computer vision tasks: motion segmentation and face clustering. Using the subspace clustering error,

Subspace clustering error $=\frac{\# \text { of misclassified points }}{\text { total } \# \text { of points }}$

as a measure of performance, we compare LRSC to state-of-the-art subspace clustering algorithms based on spectral clustering, such as LSA (Yan and Pollefeys, 2006), SCC (Chen and Lerman, 2009), LRR (Liu et al., 2010), and SSC (Elhamifar and Vidal, 2013). We choose these methods as a baseline, because they have been shown to perform very well on the above tasks, as reported in Vidal (2011). For the state-of-the-art algorithms, we use the implementations provided by their authors. The parameters of the different methods are set as shown in Table 2.

Notice that the SSC and LRR algorithms in Elhamifar and Vidal (2013) and Liu et al. (2010), respectively, apply spectral clustering to a similarity graph built from the solution of their proposed optimization programs. Specifically, SSC uses the affinity $|C|+|C|^{\top}$, while LRR uses the affinity $|C|$. However, the implementation of the SSC algorithm normalizes the columns of $C$ to be of unit $\ell_{1}$ norm. To investigate the effect of this post-processing step, we report the results for both cases of without (SSC) and with (SSC-N) the column normalization step. Also, the code of the LRR algorithm in Liu et al. (2012) applies a heuristic post-processing step to the low-rank solution prior to building the similarity graph, similar to Lauer and Schnörr (2009). Thus, we report the results for both without (LRR) and with (LRR-H) the heuristic post-processing step.

Notice also that the original published code of LRR contains the function "compacc.m" for computing the misclassification rate, which is erroneous, as noted in Elhamifar and Vidal (2013). Here, we use the correct code for computing the misclassification rate and as a result, the reported performance for LRR-H is different from the published results in Liu et al. (2010, 2012). Likewise, our results for LRSC are different from those in Favaro et al. (2011), which also used the erroneous function "compacc.m".

Finally, to have a fair comparison, since LSA and SCC need to know the number of subspaces a priori and the estimation of the number of subspaces from the eigenspectrum of the graph Laplacian in the noisy setting is often unreliable, we provide the number of subspaces as an input to all the algorithms.

\subsection{Experiments on motion segmentation}

Motion segmentation refers to the problem of clustering a set of 2D point trajectories extracted from a video sequence into groups corresponding to different rigid-body motions. Here, the data matrix $D$ is of dimension $2 F \times N$, where $N$ is the number of $2 \mathrm{D}$ trajectories and $F$ is the number of frames in the video. Under the affine 
Table 2

Parameter setup of different algorithms. $K$ is the number of nearest neighbors used by LSA to fit a local subspace around each data point, $d$ is the dimension of each subspace assumed by LSA and SCC, $\tau$ is a parameter weighting the self-expressiveness error, $\alpha$ is a parameter weighting noise, and $\gamma$ is a parameter weighting gross errors by the $\ell_{1}$ (SSC, LRSC) or $\ell_{2,1}$ (LRR) norms.

\begin{tabular}{|c|c|c|c|c|c|}
\hline Parameter & LSA & SCC & LRR & SSC & LRSC \\
\hline \multicolumn{6}{|c|}{ Motion segmentation } \\
\hline$K$ & 8 & & & & \\
\hline$d$ & 4 & 3 & & & \\
\hline$\tau$ & & & & & $420, \frac{4.5 \times 10^{4}}{\sqrt{M N}}, \frac{6 \times 10^{4}}{\sqrt{M N}}$ \\
\hline$\alpha$ & & & & $\frac{800}{\min _{i} \max _{j \neq i}\left|\boldsymbol{d}_{i} \boldsymbol{d}_{j}\right|}$ & $3000,5000, \infty$ \\
\hline$\gamma$ & & & 4 & $\infty$ & $5, \infty$ \\
\hline$\eta$ & & & & & 0.03 \\
\hline$\mu_{0}$ & & & & & 100 \\
\hline$\rho$ & & & & 1.0 & 1.1 \\
\hline \multicolumn{6}{|c|}{ Face clustering } \\
\hline$K$ & 7 & & & & \\
\hline$d$ & 5 & 9 & & & \\
\hline$\tau$ & & & & & $0.01,0.03,0.045,0.07,0.1$ \\
\hline$\alpha$ & & & & $\infty$ & $0.03,0.045,0.07,0.1, \infty$ \\
\hline$\gamma$ & & & 0.18 & $\frac{20}{\min _{i} \max _{j \neq i}\left\|\boldsymbol{d}_{j}\right\|_{1}}$ & $10^{-6}, 0.01, \infty$ \\
\hline$\eta$ & & & & & $0.03,0.04$ \\
\hline$\mu_{0}$ & & & & & 0.03 \\
\hline$\rho$ & & & & 1.0 & 1.5 \\
\hline
\end{tabular}

projection model, the $2 \mathrm{D}$ trajectories associated with a single rigidbody motion live in an affine subspace of $\mathbb{R}^{2 F}$ of dimension $d=1,2$ or 3 (Tomasi and Kanade, 1992). Therefore, the trajectories associated with $n$ different moving objects lie in a union of $n$ affine subspaces in $\mathbb{R}^{2 F}$, and the motion segmentation problem reduces to clustering a collection of point trajectories according to multiple affine subspaces. Since LRSC is designed to cluster linear subspaces, we apply LRSC to the trajectories in homogeneous coordinates, i.e., we append a constant $\eta=0.1$ and work with $2 F+1$ dimensional vectors.

We use the Hopkins155 motion segmentation database (Tron and Vidal, 2007) to evaluate the performance of LRSC against that of other algorithms. The database, which is available online at http://www.vision.jhu.edu/data/hopkins155, consists of 155 sequences of two and three motions. For each sequence, the 2D trajectories are extracted automatically with a tracker and outliers are manually removed. Fig. 2 shows some sample images with the feature points superimposed.

Table 3(a) and (b) give the average subspace clustering error obtained by different variants of LRSC on the Hopkins 155 motion segmentation database. We can see that most variants of LRSC have a similar performance. This is expected, because the trajectories are corrupted by noise, but do not have gross errors. Therefore, the Frobenius norm on the errors performs almost as well as the $\ell_{1}$ norm. However, the performance depends on the choice of the parameters. In particular, notice that choosing $\tau$ that depends on the number of motions and size of each sequence gives better results than using a fixed $\tau$.

Table 4(a) and (b) compare the best results of LRSC against the state-of-the-art results. Overall, LRSC compares favorably against LSA, SCC and LRR without post-processing of the affinity matrix. Relative to LRR with post-processing, LRSC performs worse when the data is not projected, and better when the data is projected. However, LRSC does not perform as well as either version of SSC (with or without post-processing).

Overall, we can see that even the simplest version of $\operatorname{LRSC}\left(P_{1}\right)$, whose solution can be computed in closed form, performs on par with state-of-the-art motion segmentation methods, which require solving a convex optimization problem. We also notice that LRSC has almost the same performance with or without projection.

\subsection{Experiments on face clustering}

Face clustering refers to the problem of clustering a set of face images from multiple individuals according to the identity of each individual. Here, the data matrix $D$ is of dimension $P \times N$, where $P$ is the number of pixels, and $N$ is the number of images. For a Lambertian object, the set of all images taken under all lighting conditions, but the same viewpoint and expression, forms a cone in the image space, which can be well approximated by a lowdimensional subspace (Basri and Jacobs, 2003). In practice, a few pixels deviate from the Lambertian model due to cast shadows and specularities, which can be modeled as sparse outlying entries. Therefore, the face clustering problem reduces to clustering a set of images according to multiple subspaces and corrupted by sparse gross errors.

We use the Extended Yale B database (Lee et al., 2005) to evaluate the performance of LRSC against that of state-of-the-art methods. The database includes 64 frontal face images of 38 individuals acquired under 64 different lighting conditions. Each image is cropped to $192 \times 168$ pixels. Fig. 3 shows sample images from the database. To reduce the computational cost and the memory requirements of all algorithms, we downsample the images to $48 \times 42$ pixels and treat each 2,016-dimensional vectorized image as a data point.

Following the experimental setup of Elhamifar and Vidal (2013), we divide the 38 subjects into 4 groups, where the first three groups correspond to subjects 1 to 10,11 to 20,21 to 30 , and the fourth group corresponds to subjects 31 to 38 . For each of the first three groups we consider all choices of $n \in\{2,3,5,8,10\}$ subjects and for the last group we consider all choices of $n \in\{2,3,5,8\}$. Finally, we apply all subspace clustering algorithms for each trial, i.e., each set of $n$ subjects.

In Table 5 we report Table 3 of Elhamifar and Vidal (2013). This table shows the average and median subspace clustering errors of different algorithms. The results are obtained by first applying the Robust Principal Component Analysis (RPCA) algorithm of Candès et al. (2011) to the face images of each subject and then applying different subspace clustering algorithms to the low-rank component of the data obtained by RPCA. The purpose of this experiment is to show that when there are no outliers in the data, our solution correctly identifies the subspaces. As described in Elhamifar and Vidal, 2013, notice that LSA and SCC do not perform well, even with de-corrupted data. Notice also that LRR-H does not perform well for more than 8 subjects, showing that the post processing step on the obtained low-rank coefficient matrix not always improves the result of LRR. SSC and LRSC, on the other hand, perform very well, with LRSC achieving perfect performance. In this test LRSC corresponds to $P_{5}-\mathrm{ADMM}$ with an additional term $\left\langle Y_{1}, A-A C\right\rangle$, where $Y_{1}$ is the Lagrange multiplier for the equation $A=A C$, with parameters $\mu_{0}=3 \tau_{0}=0.5\left(1.25 / \sigma_{1}(D)\right)^{2}, \gamma=0.008, \rho=1.8$. The update step for the additional Lagrange multiplier and the augmented coefficient $\tau_{k}$ is $Y_{1, k+1}=Y_{1, k}+\tau_{k}\left(A_{k+1}-A_{k+1} C_{k+1}\right)$ and $\tau_{k+1}=\rho \tau_{k}$. We also have repeated the test with our current implementations and also found the error to be consistently 0 across all subjects. In our test we preprocessed each subject with $P_{5}-$ ADMM with parameters $\eta=0, \tau=0.01, \gamma=10^{-6}$ and run for 10 iterations. Then we ran $P_{3}$ with parameters $\eta=0.04$, $\tau=0.1$ and $\alpha=0.1$.

Table 6 shows the results of applying different clustering algorithms to the original data, without first applying RPCA to each group. Notice that the performance of LSA and SCC deteriorates dramatically, showing that these methods are very sensitive to gross errors. The performance of LRR is better, but the errors are still very high, especially as the number of subjects increases. In this case, the post processing step of LRR-H does help to significantly reduce the clustering error. SSC-N, on the other hand, 

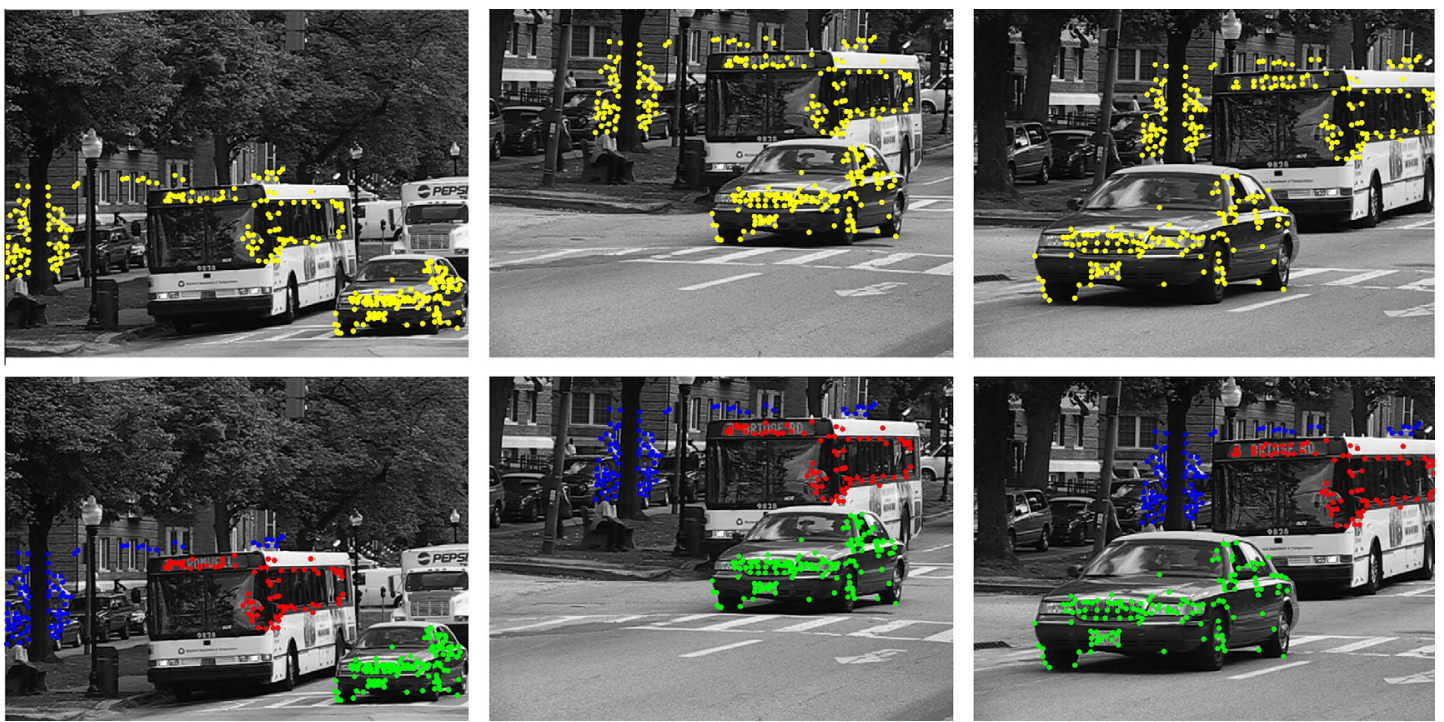

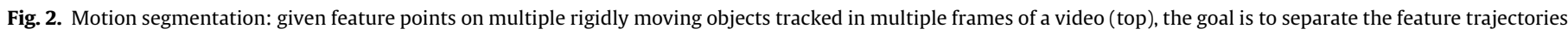
according to the moving objects (bottom).

Table 3

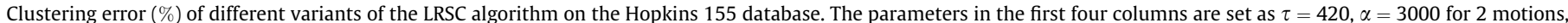

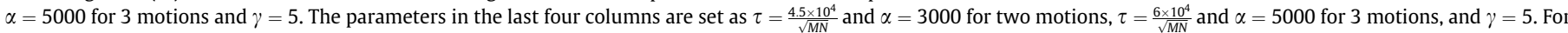
$P_{5}$-ADMM, we set $\mu_{0}=100$ and $\rho=1$.1. In all cases, we use homogeneous coordinates with $\eta=0.1$. Boldface indicates the top performing algorithm in each experiment.

\begin{tabular}{|c|c|c|c|c|c|c|c|c|}
\hline Method & $P_{1}$ & $P_{3}$ & $P_{5}-\mathrm{ADMM}$ & $P_{5}$-IPT & $P_{1}$ & $P_{3}$ & $P_{5}-\mathrm{ADMM}$ & $P_{5}$-IPT \\
\hline \multicolumn{9}{|c|}{$\begin{array}{l}\text { (a) } 2 F \text {-dimensional data points } \\
2 \text { Motions }\end{array}$} \\
\hline Mean & 3.39 & 3.27 & 3.13 & 3.27 & 2.58 & 2.57 & 2.62 & 2.57 \\
\hline Median & 0.00 & 0.00 & 0.00 & 0.00 & 0.00 & 0.00 & 0.00 & 0.00 \\
\hline \multicolumn{9}{|l|}{3 Motions } \\
\hline Mean & 7.28 & 7.29 & 7.31 & 7.29 & 6.68 & 6.64 & 6.76 & 6.67 \\
\hline Median & 2.53 & 2.53 & 2.53 & 2.53 & 1.76 & 1.76 & 1.76 & 1.76 \\
\hline \multicolumn{9}{|l|}{ All } \\
\hline Mean & 4.25 & 4.16 & 4.05 & 4.16 & 3.49 & 3.47 & 3.53 & 3.48 \\
\hline Median & 0.00 & 0.19 & 0.00 & 0.19 & 0.09 & 0.09 & 0.00 & 0.09 \\
\hline \multicolumn{9}{|c|}{$\begin{array}{l}\text { (b) } 4 n \text {-dimensional data points obtained by applying PCA to original data } \\
2 \text { Motions }\end{array}$} \\
\hline Mean & 3.19 & 3.28 & 3.93 & 3.28 & 2.59 & 2.57 & 3.43 & 2.57 \\
\hline Median & 0.00 & 0.00 & 0.00 & 0.00 & 0.00 & 0.00 & 0.00 & 0.00 \\
\hline \multicolumn{9}{|l|}{3 Motions } \\
\hline Mean & 7.27 & 7.28 & 8.07 & 7.28 & 6.64 & 6.67 & 8.13 & 6.62 \\
\hline Median & 2.54 & 2.54 & 3.76 & 2.54 & 1.76 & 1.76 & 2.30 & 1.76 \\
\hline \multicolumn{9}{|l|}{ All } \\
\hline Mean & 4.09 & 4.16 & 4.85 & 4.17 & 3.49 & 3.48 & 4.48 & 3.47 \\
\hline Median & 0.19 & 0.19 & 0.21 & 0.19 & 0.19 & 0.09 & 0.19 & 0.00 \\
\hline
\end{tabular}

performs very well, achieving a clustering error of about $10 \%$ for 10 subjects. Table 7 shows the performance of different variants of LRSC on the same data. $P_{1}$ has the best performance for 2 and 3 subjects, and the worst performance for 5,8 and 10 subjects. All other variants of LRSC have similar performance, perhaps with $P_{5}$-IPT being the best. Overall, LRSC performs better than LSA, SCC and LRR, and worse than LRR-H and SSC-N.

Finally, Fig. 4 shows the average computational time of each algorithm as a function of the number of subjects (or equivalently the number of data points). Note that the computational time of SCC is drastically higher than other algorithms. This comes from the fact that the complexity of SCC increases exponentially in the dimension of the subspaces, which in this case is $d=9$. On the other hand, SSC, LRR and LRSC use fast and efficient convex optimization techniques which keep their computational time lower than that of other algorithms. Overall, LRR and LRSC are the fastest methods.

\section{Discussion and conclusion}

We have proposed a new algorithm for clustering data drawn from a union of subspaces and corrupted by noise/gross errors. Our approach was based on solving a non-convex optimization problem whose solution provides an affinity matrix for spectral clustering. Our key contribution was to show that important particular cases of our formulation can be solved in closed form by applying a polynomial thresholding operator to the SVD of the data. A drawback of our approach to be addressed in the future is the need to tune the parameters of our cost function. Further research is also needed to understand the correctness of the resulting affinity matrix in the presence of noise and corruptions. Finally, all existing methods decouple the learning of the affinity from the segmentation of the data. Further research is needed to integrate these two steps into a single objective. 
Table 4

Clustering error (\%) on the Hopkins 155 database. Boldface indicates the top performing algorithm in each experiment.

\begin{tabular}{|c|c|c|c|c|c|c|c|}
\hline Method & LSA & SCC & LRR & LRR-H & SSC & SSC-N & LRSC \\
\hline \multicolumn{8}{|c|}{$\begin{array}{l}\text { (a) } 2 F \text {-dimensional data points } \\
2 \text { Motions }\end{array}$} \\
\hline Mean & 4.23 & 2.89 & 4.10 & 2.13 & 2.07 & 1.52 & 2.57 \\
\hline Median & 0.56 & 0.00 & 0.22 & 0.00 & 0.00 & 0.00 & 0.00 \\
\hline \multicolumn{8}{|l|}{3 Motions } \\
\hline Mean & 7.02 & 8.25 & 9.89 & 4.03 & 5.27 & 4.40 & 6.64 \\
\hline Median & 1.45 & 0.24 & 6.22 & 1.43 & 0.40 & 0.56 & 1.76 \\
\hline \multicolumn{8}{|l|}{ All } \\
\hline Mean & 4.86 & 4.10 & 5.41 & 2.56 & 2.79 & 2.18 & 3.47 \\
\hline Median & 0.89 & 0.00 & 0.53 & 0.00 & 0.00 & 0.00 & 0.09 \\
\hline \multicolumn{8}{|c|}{ (b) $4 n$-dimensional data points obtained by applying PCA to original data } \\
\hline \multicolumn{8}{|c|}{2 Motions } \\
\hline Mean & 3.61 & 3.04 & 4.83 & 3.41 & 2.14 & 1.83 & 2.57 \\
\hline Median & 0.51 & $\mathbf{0 . 0 0}$ & 0.26 & 0.00 & $\mathbf{0 . 0 0}$ & $\mathbf{0 . 0 0}$ & 0.00 \\
\hline \multicolumn{8}{|l|}{3 Motions } \\
\hline Mean & 7.65 & 7.91 & 9.89 & 4.86 & 5.29 & 4.40 & 6.62 \\
\hline Median & 1.27 & 1.14 & 6.22 & 1.47 & 0.40 & 0.56 & 1.76 \\
\hline \multicolumn{8}{|l|}{ All } \\
\hline Mean & 4.52 & 4.14 & 5.98 & 3.74 & 2.85 & 2.41 & 3.47 \\
\hline Median & 0.57 & 0.00 & 0.59 & 0.00 & 0.00 & 0.00 & 0.00 \\
\hline
\end{tabular}

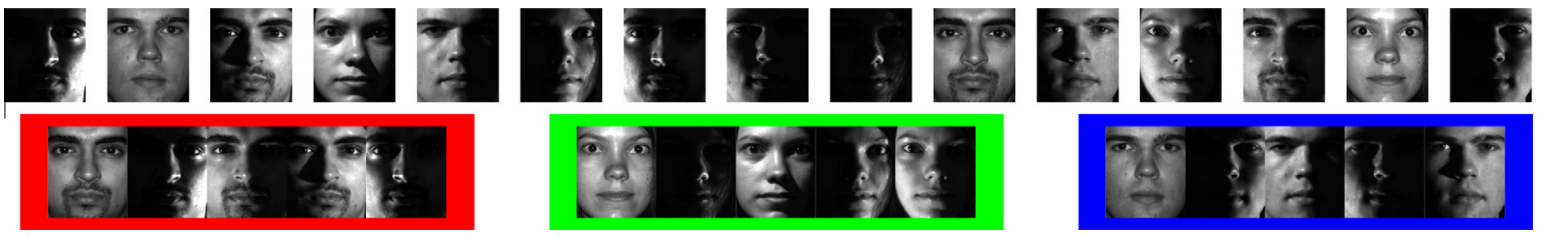

Fig. 3. Face clustering: given face images of multiple subjects (top), the goal is to find images that belong to the same subject (bottom).

\section{Appendix A. The Von Neumann trace inequality}

This appendix reviews two matrix product inequalities, which we will use later in our derivations.

Lemma 1 (Von Neumann's inequality). For any $m \times n$ real valued matrices $X$ and $Y$,

$\operatorname{trace}\left(X^{\top} Y\right) \leqslant \sum_{i=1}^{n} \sigma_{i}(X) \sigma_{i}(Y)$ where $\sigma_{1}(X) \geqslant \sigma_{2}(X) \geqslant \cdots \geqslant 0$ and $\sigma_{1}(Y) \geqslant \sigma_{2}(Y) \geqslant \cdots \geqslant 0$ are the descending singular values of $X$ and $Y$ respectively. The case of equality occurs if and only if it is possible to find unitary matrices $U_{X}$ and $V_{X}$ that simultaneously singular value-decompose $X$ and $Y$ in the sense that

$X=U_{X} \Sigma_{X} V_{X}^{\top} \quad$ and $\quad Y=U_{X} \Sigma_{Y} V_{X}^{\top}$,

where $\Sigma_{X}$ and $\Sigma_{Y}$ denote the $m \times n$ diagonal matrices with the singular values of $X$ and $Y$, respectively, down in the diagonal.

Table 6

Clustering error (\%) of different algorithms on the Extended Yale B database without pre-processing the data. Boldface indicates the top performing algorithm in each experiment.

\begin{tabular}{llllll}
\hline Method & LSA & SCC & LRR & LRR-H & SSC-N \\
\hline 2 Subjects & & & & & \\
Mean & 32.80 & 16.62 & 9.52 & 2.54 & $\mathbf{1 . 8 6}$ \\
Median & 47.66 & 7.82 & 5.47 & 0.78 & $\mathbf{0 . 0 0}$ \\
3 Subjects & & & & & \\
Mean & 52.29 & 38.16 & 19.52 & 4.21 & $\mathbf{3 . 1 0}$ \\
Median & 50.00 & 39.06 & 14.58 & 2.60 & $\mathbf{1 . 0 4}$ \\
5 Subjects & & & & & \\
Mean & 58.02 & 58.90 & 34.16 & 6.90 & $\mathbf{4 . 3 1}$ \\
Median & 56.87 & 59.38 & 35.00 & 5.63 & $\mathbf{2 . 5 0}$ \\
8 Subjects & & & & & \\
Mean & 59.19 & 66.11 & 41.19 & 14.34 & $\mathbf{5 . 8 5}$ \\
Median & 58.59 & 64.65 & 43.75 & 10.06 & $\mathbf{4 . 4 9}$ \\
10 Subjects & & & & & \\
Mean & 60.42 & 73.02 & 38.85 & 22.92 & $\mathbf{1 0 . 9 4}$ \\
Median & 57.50 & 75.78 & 41.09 & 23.59 & $\mathbf{5 . 6 3}$ \\
\hline
\end{tabular}

Please cite this article in press as: Vidal, R., Favaro, P. Low rank subspace clustering (LRSC). Pattern Recognition Lett. (2013), http://dx.doi.org/10.1016/ j.patrec.2013.08.006 
Table 7

Clustering error (\%) of our algorithms on the Extended Yale B database without preprocessing the data. The parameters of LRSC $P_{1}$ and $P_{3}$ are set as $\tau=0.045$, and $\alpha=0.045$, while the coefficient for homogeneous coordinates is set as $\eta=0.03$. $P_{5}$ ADMM was run for 20 iterations, with parameters $\tau=0.03, \gamma=0.01, \eta=0.03$, $\mu_{0}=0.03$ and $\rho=1.5$. $P_{5}$-IPT was run for 10 iterations, with parameters $\alpha=0.07$, $\tau=0.07, \gamma=0.01$, and $\eta=0.03$.

\begin{tabular}{llll}
\hline$P_{1}$ & $P_{3}$ & $P_{5}$-ADMM & $P_{5}$-IPT \\
\hline 2 Subjects & & & \\
3.15 & 4.28 & 3.27 & 4.52 \\
2.34 & 3.91 & 2.34 & 3.91 \\
3 Subjects & & & \\
4.71 & 6.23 & 5.46 & 5.80 \\
4.17 & 5.73 & 4.17 & 5.73 \\
5 Subjects & & & \\
13.06 & 14.31 & 14.31 & 8.97 \\
8.44 & 7.81 & 8.44 & 7.81 \\
8 Subjects & & & 22.61 \\
26.83 & 23.73 & 29.91 & 26.46 \\
28.71 & 27.83 & 30.27 & \\
10 Subjects & & & 29.01 \\
35.89 & 31.25 & 36.88 & 26.88 \\
34.84 & 27.65 & 36.25 & \\
\hline
\end{tabular}

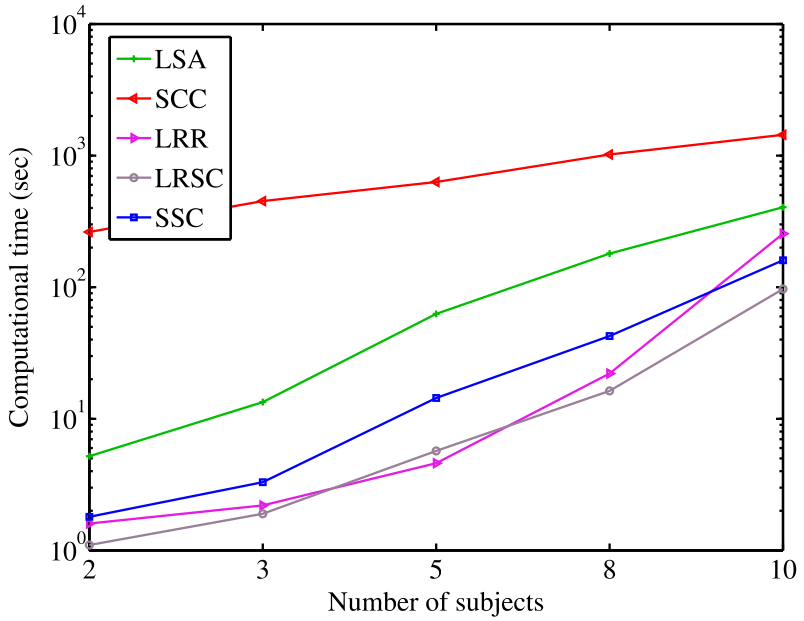

Fig. 4. Average computational time (s) of the algorithms on the Extended Yale B database as a function of the number of subjects.

Proof. See Mirsky (1975).

Lemma 2. For any $n \times n$ real valued, symmetric positive definite matrices $X$ and $Z$,

$\operatorname{trace}(X Z) \geqslant \sum_{i=1}^{n} \sigma_{i}(X) \sigma_{n-i+1}(Z)$

where $\sigma_{1}(X) \geqslant \sigma_{2}(X) \geqslant \cdots \geqslant 0$ and $\sigma_{1}(Z) \geqslant \sigma_{2}(Z) \geqslant \cdots \geqslant 0$ are the descending singular values of $X$ and $Z$, respectively. The case of equality occurs if and only if it is possible to find a unitary matrix $U_{X}$ that simultaneously singular value-decomposes $X$ and $Z$ in the sense that

$X=U_{X} \Sigma_{X} U_{X}^{\top} \quad$ and $\quad Z=U_{X} \Pi \Sigma_{Z} \Pi^{\top} U_{X}^{\top}$,

where $\Sigma_{X}$ and $\Sigma_{Z}$ denote the $n \times n$ diagonal matrices with the singular values of $X$ and $Z$, respectively, down in the diagonal in descending order, and $\Pi$ is a permutation matrix such that $\Pi \Sigma_{Z} \Pi^{\top}$ contains the singular values of $Z$ in the diagonal in ascending order.
Proof. Let $\quad Y=\lambda I-Z, \quad$ where $\quad \lambda \geqslant\|Z\|_{2}$. Then $\operatorname{trace}(X Y)=\operatorname{trace}(X(\lambda I-Z))=\lambda \operatorname{trace}(X)-\operatorname{trace}(X Z)$. Also,

$$
\begin{aligned}
\operatorname{trace}(X Y) & \leqslant \sum_{i=1}^{n} \sigma_{i}(X) \sigma_{i}(Y)=\sum_{i=1}^{n} \sigma_{i}(X) \sigma_{i}(\lambda I-Z) \\
& =\sum_{i=1}^{n} \sigma_{i}(X)\left(\lambda-\sigma_{n-i+1}(Z)\right) \\
& =\lambda \operatorname{trace}(X)-\sum_{i=1}^{n} \sigma_{i}(X) \sigma_{n-i+1}(Z)
\end{aligned}
$$

It follows from Lemma 1 that $\operatorname{trace}(X Z) \geqslant \sum_{i=1}^{n} \sigma_{i}(X) \sigma_{n-i+1}(Z)$, as claimed. Moreover, the equality is achieved if an only if there exists a matrix $U_{X}$ (recall that $X$ and $Z$ are symmetric) such that $X=U_{X} \Sigma_{X} U_{X}^{\top}$ and $Y=U_{X} \Sigma_{Y} U_{X}^{\top}$. Therefore,

$$
\begin{aligned}
Z & =\lambda I-Y=\lambda I-U_{X} \Sigma_{Y} U_{X}^{\top}=\lambda I-U_{X} \Sigma_{\lambda I-Z} U_{X}^{\top} \\
& =\lambda I-U_{X}\left(\lambda I-\Pi \Sigma_{Z} \Pi^{\top}\right) U_{X}^{\top}=U_{X} \Pi \Sigma_{Z} \Pi^{\top} U_{X}^{\top}
\end{aligned}
$$

as claimed.

\section{Appendix B. Proofs of the main theorems}

Theorem 6. Let $A=U \Lambda V^{\top}$ be the SVD of $A$, where the diagonal entries of $\Lambda=\operatorname{diag}\left(\left\{\lambda_{i}\right\}\right)$ are the singular values of $A$ in decreasing order. The optimal solution to $P_{1}$ is

$C=V \mathcal{P}_{\tau}(\Lambda) V^{\top}=V_{1}\left(I-\frac{1}{\tau} \Lambda_{1}^{-2}\right) V_{1}^{\top}$,

where the operator $\mathcal{P}_{\tau}$ acts on the diagonal entries of $\Lambda$ as

$\mathcal{P}_{\tau}(x)= \begin{cases}1-\frac{1}{\tau x^{2}} & x>1 / \sqrt{\tau} \\ 0 & x \leqslant 1 / \sqrt{\tau},\end{cases}$

and $U=\left[U_{1} U_{2}\right], \Lambda=\operatorname{diag}\left(\Lambda_{1}, \Lambda_{2}\right)$ and $V=\left[V_{1} V_{2}\right]$ are partitioned according to the sets $\mathbf{I}_{1}=\left\{i: \lambda_{i}>1 / \sqrt{\tau}\right\}$ and $\mathbf{I}_{2}=\left\{i: \lambda_{i} \leqslant 1 / \sqrt{\tau}\right\}$. Moreover, the optimal value is

$\Phi_{\tau}(A) \doteq \sum_{i \in \mathbf{I}_{1}}\left(1-\frac{1}{2 \tau} \lambda_{i}^{-2}\right)+\frac{\tau}{2} \sum_{i \in \mathbf{I}_{2}} \lambda_{i}^{2}$

Proof. Let $A=U \Lambda V^{\top}$ be the SVD of $A$ and $C=U_{C} \Delta U_{C}^{\top}$ be the eigenvalue decomposition (EVD) of $C$. The cost function of $P_{1}$ reduces to

$$
\begin{gathered}
\left\|U_{C} \Delta U_{C}^{\top}\right\|_{*}+\frac{\tau}{2}\left\|U \Lambda V^{\top}\left(I-U_{C} \Delta U_{C}^{\top}\right)\right\|_{F}^{2} \\
=\|\Delta\|_{*}+\frac{\tau}{2}\left\|\Lambda V^{\top} U_{C}(I-\Delta) U_{C}^{\top}\right\|_{F}^{2} \\
=\|\Delta\|_{*}+\frac{\tau}{2}\|\Lambda W(I-\Delta)\|_{F}^{2},
\end{gathered}
$$

where $W=V^{\top} U_{C}$. To minimize this cost with respect to $W$, we only need to consider the last term of the cost function, i.e.,

$\|\Lambda W(I-\Delta)\|_{F}^{2}=\operatorname{trace}\left((I-\Delta)^{2} W^{\top} \Lambda^{2} W\right)$.

Applying Lemma 2 to $X=W(I-\Delta)^{2} W^{\top}$ and $Z=\Lambda^{2}$, we obtain that for all unitary matrices $W$

$\min _{W} \operatorname{trace}\left((I-\Delta)^{2} W^{\top} \Lambda^{2} W\right)=\sum_{i=1}^{N} \sigma_{i}\left((I-\Delta)^{2}\right) \sigma_{n-i+1}\left(\Lambda^{2}\right)$,

where the minimum is achieved by a permutation matrix $W=\Pi^{\top}$ that sorts the diagonal entries of $\Lambda^{2}$ in ascending order, i.e., the diagonal entries of $\Pi \Lambda^{2} \Pi^{\top}$ are in ascending order.

Let the ith largest entry of $(I-\Delta)^{2}$ and $\Lambda^{2}$ be, respectively, $\left(1-\delta_{i}\right)^{2}=\sigma_{i}\left((I-\Delta)^{2}\right)$ and $v_{n-i+1}^{2}=\lambda_{i}^{2}=\sigma_{i}\left(\Lambda^{2}\right)$. Then 
$\min _{W}\|\Delta\|_{*}+\frac{\tau}{2}\|\Lambda W(I-\Delta)\|_{F}^{2}=\sum_{i=1}^{N}\left|\delta_{i}\right|+\frac{\tau}{2} \sum_{i=1}^{N} v_{i}^{2}\left(1-\delta_{i}\right)^{2}$.

To find the optimal $\Delta$, we can solve for each $\delta_{i}$ independently as $\delta_{i}=\operatorname{argmin}_{\delta}|\delta|+\frac{\tau}{2} v_{i}^{2}(1-\delta)^{2}$. As shown, e.g., by Parikh and Boyd (2013), the solution to this problem can be found in closed form using the shrinkage-thresholding operator in (7), which gives

$\delta_{i}=\mathcal{S}_{\frac{1}{\tau v_{i}^{2}}}(1)=\left\{\begin{array}{ll}1-\frac{1}{\tau v_{i}^{2}} & v_{i}>1 / \sqrt{\tau} \\ 0 & v_{i} \leqslant 1 / \sqrt{\tau}\end{array}\right.$.

Then, $\delta_{i}=\mathcal{P}_{\tau}\left(\lambda_{n-i+1}\right)$, which can be compactly written as $\Delta=\Pi \mathcal{P}_{\tau}(\Lambda) \Pi^{\top}$. Therefore,

$\Pi^{\top} \Delta \Pi=\mathcal{P}_{\tau}(\Lambda)=\left[\begin{array}{cc}I-\frac{1}{\tau} \Lambda_{1}^{-2} & 0 \\ 0 & 0\end{array}\right]$,

where $\Lambda=\operatorname{diag}\left(\Lambda_{1}, \Lambda_{2}\right)$ is partitioned according to the sets $\mathbf{I}_{1}=\left\{i: \lambda_{i}>1 / \sqrt{\tau}\right\}$ and $\mathbf{I}_{2}=\left\{i: \lambda_{i} \leqslant 1 / \sqrt{\tau}\right\}$.

To find the optimal $W$, notice from Lemma 2 that the equality $\operatorname{trace}\left((I-\Delta)^{2} W^{\top} \Lambda^{2} W\right)=\sum_{i=1}^{N}\left(1-\delta_{i}\right)^{2} \lambda_{n-i+1}^{2}$ is achieved if and only if there exists a unitary matrix $U_{X}$ such that

$(I-\Delta)^{2}=U_{X}(I-\Delta)^{2} U_{X}^{\top}$ and $W^{\top} \Lambda^{2} W=U_{X} \Pi \Lambda^{2} \Pi^{\top} U_{X}^{\top}$.

Since the SVD of a matrix is unique up to the sign of the singular vectors associated with different singular values and up to a rotation and sign of the singular vectors associated with repeated singular values, we conclude that $U_{X}=I$ up to the aforementioned ambiguities of the SVD of $(I-\Delta)^{2}$. Likewise, we have that $W^{\top}=U_{X} \Pi$ up to the aforementioned ambiguities of the SVD of $\Lambda^{2}$. Now, if $\Lambda^{2}$ has repeated singular values, then $(I-\Delta)^{2}$ has repeated eigenvalues at the same locations. Therefore, $W^{\top}=U_{X} \Pi=\Pi$ up to a block-diagonal transformation, where each block is an orthonormal matrix that corresponds to a repeated singular value of $\Delta$. Nonetheless, even though $W$ may not be unique, the matrix $C$ is always unique and equal to

$$
\begin{aligned}
C & =U_{C} \Delta U_{C}^{\top}=V W \Delta W^{\top} V^{\top}=V \Pi^{\top} \Delta \Pi V^{\top} \\
& =\left[\begin{array}{ll}
V_{1} & V_{2}
\end{array}\right]\left[\begin{array}{cc}
I-\frac{1}{\tau} \Lambda_{1}^{-2} & 0 \\
0 & 0
\end{array}\right]\left[\begin{array}{ll}
V_{1} & V_{2}
\end{array}\right]^{\top}=V_{1}\left(I-\frac{1}{\tau} \Lambda_{1}^{-2}\right) V_{1}^{\top},
\end{aligned}
$$

as claimed.

Finally, the optimal $C$ is such that $A C=U_{1}\left(\Lambda_{1}-\frac{1}{\tau} \Lambda_{1}^{-1}\right) V_{1}^{\top}$ and $A-A C=U_{2} \Lambda_{2} V_{2}^{\top}+\frac{1}{\tau} U_{1} \Lambda_{1} V_{1}^{\top}$. This shows (22), because

$$
\|C\|_{*}+\frac{\tau}{2}\|A-A C\|_{F}^{2}=\sum_{i \in \mathbf{I}_{1}}\left(1-\frac{1}{\tau} \lambda_{i}^{-2}\right)+\frac{\tau}{2}\left(\sum_{i \in \mathbf{I}_{1}} \frac{\lambda_{i}^{-2}}{\tau^{2}}+\sum_{i \in \mathbf{I}_{2}} \lambda_{i}^{2}\right),
$$

as claimed.

Theorem 3. Let $D=U \Sigma V^{\top}$ be the SVD of the data matrix $D$. The optimal solutions to $P_{3}$ are of the form

$A=U \Lambda V^{\top}$ and $C=V \mathcal{P}_{\tau}(\Lambda) V^{\top}$,

where each entry of $\Lambda=\operatorname{diag}\left(\lambda_{1}, \ldots, \lambda_{n}\right)$ is obtained from each entry of $\Sigma=\operatorname{diag}\left(\sigma_{1}, \ldots, \sigma_{n}\right)$ as the solutions to

$$
\sigma=\psi(\lambda) \doteq \begin{cases}\lambda+\frac{1}{\alpha \tau} \lambda^{-3} & \text { if } \lambda>1 / \sqrt{\tau} \\ \lambda+\frac{\tau}{\alpha} \lambda & \text { if } \lambda \leqslant 1 / \sqrt{\tau}\end{cases}
$$

that minimize

$$
\phi(\lambda, \sigma) \doteq \frac{\alpha}{2}(\sigma-\lambda)^{2}+\left\{\begin{array}{ll}
1-\frac{1}{2 \tau} \lambda^{-2} & \lambda>1 / \sqrt{\tau} \\
\frac{\tau}{2} \lambda^{2} & \lambda \leqslant 1 / \sqrt{\tau}
\end{array} .\right.
$$

Proof. The proof of this result will be done in two steps. First, we will show that the optimal $C$ can be computed in closed form from the SVD of $A$. Second, we will show that the optimal $A$ can be obtained in closed form from the SVD of $D$.

A closed-form solution for $C$. Notice that when $A$ is fixed, $P_{3}$ reduces to $P_{1}$. Therefore, it follows from Theorem 1 that the optimal solution for $C$ is $C=V \mathcal{P}_{\tau}(\Lambda) V^{\top}$, where $A=U \Lambda V^{\top}$ is the SVD of $A$. Moreover, it follows from (22) that if we replace the optimal $C$ into the cost of $P_{3}$, then $P_{3}$ is equivalent to

$\min _{A} \Phi_{\tau}(A)+\frac{\alpha}{2}\|D-A\|_{F}^{2}$

A closed-form solution for $A$. Let $D=U \Sigma V^{\top}$ and $A=U_{A} \Lambda V_{A}^{\top}$ be the SVDs of $D$ and $A$, respectively. Then,

$$
\begin{aligned}
\| D & -A\left\|_{F}^{2}=\right\| U \Sigma V^{\top}-U_{A} \Lambda V_{A}^{\top} \|_{F}^{2}, \\
& =\|\Sigma\|_{F}^{2}-2 \operatorname{trace}\left(V \Sigma U^{\top} U_{A} \Lambda V_{A}^{\top}\right)+\|\Lambda\|_{F}^{2}, \\
& =\|\Sigma\|_{F}^{2}-2 \operatorname{trace}\left(\Sigma W_{1} \Lambda W_{2}^{\top}\right)+\|\Lambda\|_{F}^{2},
\end{aligned}
$$

where $W_{1}=U^{\top} U_{A}$ and $W_{2}=V^{\top} V_{A}$. Therefore, the minimization over $A$ in (B.15) can be carried out by minimizing first with respect to $W_{1}$ and $W_{2}$ and then with respect to $\Lambda$.

The minimization over $W_{1}$ and $W_{2}$ is equivalent to

$\max _{W_{1}, W_{2}} \operatorname{trace}\left(\Sigma W_{1} \Lambda W_{2}^{\top}\right)$.

By letting $X=\Sigma$ and $Y=W_{1} \Lambda W_{2}^{\top}$ in Lemma 1, we obtain

$\max _{W_{1}, W_{2}} \operatorname{trace}\left(\Sigma W_{1} \Lambda W_{2}^{\top}\right)=\sum_{i=1}^{n} \sigma_{i}(\Sigma) \sigma_{i}(\Lambda)=\sum_{i=1}^{n} \sigma_{i} \lambda_{i}$.

Moreover, the maximum is achieved if and only if there exist orthogonal matrices $U_{W}$ and $V_{W}$ such that

$\Sigma=U_{W} \Sigma V_{W}^{\top}$ and $W_{1} \Lambda W_{2}^{\top}=U_{W} \Lambda V_{W}^{\top}$.

Hence, the optimal solutions are $W_{1}=U_{W}=I$ and $W_{2}=V_{W}=I$ up to a unitary transformation that accounts for the sign and rotational ambiguities of the singular vectors of $\Sigma$. This means that $A$ and $D$ have the same singular vectors, i.e., $U_{A}=U$ and $V_{A}=V$, and that $\|D-A\|_{F}^{2}=\left\|U(\Sigma-\Lambda) V^{\top}\right\|_{F}^{2}=\|\Sigma-\Lambda\|_{F}^{2}$. By substituting this expression for $\|D-A\|_{F}^{2}$ into (B.15), we obtain

$\min _{\Lambda} \sum_{i \in \mathbf{I}_{1}}\left(1-\frac{1}{2 \tau} \lambda_{i}^{-2}\right)+\frac{\tau}{2} \sum_{i \in \mathbf{I}_{2}} \lambda_{i}^{2}+\frac{\alpha}{2} \sum_{i}\left(\sigma_{i}-\lambda_{i}\right)^{2}$,

where $\mathbf{I}_{1}=\left\{i: \lambda_{i}>1 / \sqrt{\tau}\right\}$ and $\mathbf{I}_{2}=\left\{i: \lambda_{i} \leqslant 1 / \sqrt{\tau}\right\}$.

It follows from the above equation that the optimal $\lambda_{i}$ can be obtained independently for each $\sigma_{i}$ by minimizing the $i$ th term of the above summation, which is of the form $\phi(\lambda, \sigma)$ in (29). The first order derivative of $\phi$ is given by

$\frac{\partial \phi}{\partial \lambda}=\alpha(\lambda-\sigma)+ \begin{cases}\frac{1}{\tau} \lambda^{-3} & \lambda>1 / \sqrt{\tau} \\ \tau \lambda & \lambda \leqslant 1 / \sqrt{\tau}\end{cases}$

Therefore, the optimal $\lambda$ 's can be obtained as the solutions of the nonlinear equation $\sigma=\psi(\lambda)$ in (28) that minimize (29). This completes the proof of Theorem 3.

Theorem 4. There exists a $\sigma_{*}>0$ such that the solutions to (28) that minimize (29) can be computed as

$\lambda=\mathcal{P}_{\alpha, \tau}(\sigma) \doteq \begin{cases}\beta_{1}(\sigma) & \text { if } \sigma \leqslant \sigma_{*} \\ \beta_{3}(\sigma) & \text { if } \sigma>\sigma_{*}\end{cases}$

where $\beta_{1}(\sigma) \doteq \frac{\alpha}{\alpha+\tau} \sigma$ and $\beta_{3}(\sigma)$ is the real root of the polynomial 
$p(\lambda)=\lambda^{4}-\sigma \lambda^{3}+\frac{1}{\alpha \tau}=0$

that minimizes (29). If $3 \tau \leqslant \alpha$, the solution for $\lambda$ is unique and

$\sigma_{*}=\psi\left(\frac{1}{\sqrt{\tau}}\right)=\frac{\alpha+\tau}{\alpha \sqrt{\tau}}$.

If $3 \tau>\alpha$, the solution for $\lambda$ is unique, except when $\sigma$ satisfies

$\phi\left(\beta_{1}(\sigma), \sigma\right)=\phi\left(\beta_{3}(\sigma), \sigma\right)$,

and $\sigma_{*}$ must lie in the range

$\frac{4}{3} \sqrt[4]{\frac{3}{\alpha \tau}}<\sigma_{*}<\frac{\alpha+\tau}{\alpha \sqrt{\tau}}$.

Proof. When $3 \tau \leqslant \alpha$, the solution to $\sigma=\psi(\lambda)$ that minimizes $\phi(\lambda, \sigma)$ is unique, as illustrated in Fig. 1(b). This is because

$\frac{\partial^{2} \phi}{\partial \lambda^{2}}= \begin{cases}\alpha-\frac{3}{\tau} \lambda^{-4} & \lambda>1 / \sqrt{\tau} \\ \alpha+\tau & \lambda \leqslant 1 / \sqrt{\tau}\end{cases}$

is strictly positive, hence $\phi$ is a strictly convex function of $\lambda$. When $\lambda \leqslant \frac{1}{\sqrt{\tau}}$, the unique solution to $\sigma=\psi(\lambda)$ is given by

$\lambda=\beta_{1}(\sigma) \doteq \frac{\alpha}{\alpha+\tau} \sigma$.

From this equation we immediately conclude that $\sigma_{*}=\frac{\alpha+\tau}{\alpha \sqrt{\tau}}$. Now, if $\lambda>\frac{1}{\sqrt{\tau}}$, then $\lambda$ must be one of the real solutions of the polynomial in (B.23). Since $3 \tau \leqslant \alpha$, this polynomial has a unique real root with multiplicity 2 , which we denote as $\beta_{3}(\sigma)$.

When $3 \tau>\alpha$, we have $\lambda=\beta_{1}(\sigma)$ if $\sigma<\theta_{1} \doteq \frac{4}{3} \sqrt[4]{\frac{3}{\alpha \tau}}$ and $\lambda=\beta_{3}(\sigma)$ if $\sigma>\theta_{3} \doteq \frac{\alpha+\tau}{\alpha \sqrt{\tau}}$, as illustrated in Fig. 1(c). However, if $\theta_{1} \leqslant \sigma \leqslant \theta_{3}$ there could be up to three solutions for $\lambda$. The first candidate solution is $\beta_{1}(\sigma)$. The remaining two candidate solutions $\beta_{2}(\sigma)$ and $\beta_{3}(\sigma)$ are the two real roots of the polynomial in (B.23), with $\beta_{2}(\sigma)$ being the smallest and $\beta_{3}(\sigma)$ being the largest root. The other two roots of $p$ are complex. Out of the three candidate solutions, $\beta_{1}(\sigma)$ and $\beta_{3}(\sigma)$ correspond to a minimum and $\beta_{2}(\sigma)$ corresponds to a maximum, because

$\beta_{1}(\sigma) \leqslant 1 / \sqrt{\tau}, \quad \beta_{2}(\sigma)<\sqrt[4]{\frac{3}{\alpha \tau}}$ and $\beta_{3}(\sigma)>\sqrt[4]{\frac{3}{\alpha \tau}}$,

and so $\frac{\partial^{2} \phi}{\partial 2^{2}}$ is positive for $\beta_{1}$, negative for $\beta_{2}$ and positive for $\beta_{3}$. Therefore, except when $\sigma$ is such that (B.25) holds true, the solution to $\sigma=\psi(\lambda)$ that minimizes (29) is unique and equal to

$\lambda= \begin{cases}\beta_{1}(\sigma) & \text { if } \phi\left(\beta_{1}(\sigma), \sigma\right)<\phi\left(\beta_{3}(\sigma), \sigma\right) \\ \beta_{3}(\sigma) & \text { if } \phi\left(\beta_{1}(\sigma), \sigma\right)>\phi\left(\beta_{3}(\sigma), \sigma\right) .\end{cases}$

To show that $\lambda$ can be obtained as in (B.22), we need show that there exists a $\sigma_{*}>0$ such that $\phi\left(\beta_{1}(\sigma), \sigma\right)<\phi\left(\beta_{3}(\sigma), \sigma\right)$ for $\sigma<\sigma_{*}$ and $\phi\left(\beta_{1}(\sigma), \sigma\right)>\phi\left(\beta_{3}(\sigma), \sigma\right)$ for $\sigma>\sigma_{*}$. Because of the intermediate value theorem, it is sufficient to show that

$f(\sigma)=\phi\left(\beta_{1}(\sigma), \sigma\right)-\phi\left(\beta_{3}(\sigma), \sigma\right)$

is continuous and increasing for $\sigma \in\left[\theta_{1}, \theta_{3}\right]$, negative at $\theta_{1}$ and positive at $\theta_{3}$, so that there is a $\sigma_{*} \in\left(\theta_{1}, \theta_{3}\right)$ such that $f\left(\sigma_{*}\right)=0$. The function $f$ is continuous in $\left[\theta_{1}, \theta_{3}\right]$, because (a) $\phi$ is a continuous function of $(\lambda, \sigma)$, (b) the roots of a polynomial ( $\beta_{1}$ and $\left.\beta_{2}\right)$ vary continuously as a function of the coefficients $(\sigma)$ and (c) the composition of two continuous functions is continuous. Also, $f$ is increasing in $\left[\theta_{1}, \theta_{3}\right]$, because

$$
\begin{aligned}
\frac{d f}{d \sigma} & =\left.\frac{\partial \phi}{\partial \lambda}\right|_{\left(\beta_{1}, \sigma\right)} \frac{d \beta_{1}}{d \sigma}+\left.\frac{\partial \phi}{\partial \sigma}\right|_{\left(\beta_{1}, \sigma\right)}-\left.\frac{\partial \phi}{\partial \lambda}\right|_{\left(\beta_{3}, \sigma\right)} \frac{d \beta_{3}}{d \sigma}-\left.\frac{\partial \phi}{\partial \sigma}\right|_{\left(\beta_{3}, \sigma\right)} \\
& =0+\alpha\left(\sigma-\beta_{1}\right)-0-\alpha\left(\sigma-\beta_{3}\right)=\alpha\left(\beta_{3}-\beta_{1}\right)>0 .
\end{aligned}
$$

Now, notice from Fig. 1(c) that when $\sigma<\theta_{1}$ the optimal solution is $\lambda=\beta_{1}$. When $\sigma=\theta_{1}, \quad \beta_{1}=\frac{4 \alpha}{3(\alpha+\tau)} \sqrt[4]{\frac{3}{\alpha \tau}} \quad$ is a minimum and $\beta_{2}=\beta_{3}=\sqrt[4]{\frac{3}{\alpha \tau}}$ is an inflection point, thus the optimal solution is $\lambda=\beta_{1}{ }^{1}{ }^{1}$ When $\sigma>\theta_{3}$, the optimal solution is $\beta_{3}$. Finally, when $\sigma=\theta_{3}, \beta_{1}=\beta_{2}=\frac{1}{\sqrt{\tau}}$ is a maximum and $\beta_{3}$ is a minimum, thus the optimal solution is $\lambda=\beta_{3}$. Therefore, the threshold for $\sigma$ must lie in the range

$\overline{3} \sqrt[4]{\frac{3}{\alpha \tau}}<\sigma_{*}<\frac{\alpha+\tau}{\alpha \sqrt{\tau}}$

\section{References}

Agarwal, P., Mustafa, N., 2004. k-Means projective clustering. In: ACM Symposium on Principles of database systems.

Basri, R., Jacobs, D., 2003. Lambertian reflection and linear subspaces. IEEE Transactions on Pattern Analysis and Machine Intelligence 25 (3), 218-233.

Boult, T., Brown, L., 1991. Factorization-based segmentation of motions. In: IEEE Workshop on Motion Understanding, pp. 179-186.

Bradley, P.S., Mangasarian, O.L., 2000. k-plane clustering. Journal of Global Optimization 16 (1), 23-32.

Cai, J.-F., Candés, E.J., Shen, Z., 2008. A singular value thresholding algorithm for matrix completion. SIAM Journal of Optimization 20 (4), 1956-1982.

Candès, E., Li, X., Ma, Y., Wright, J., 2011. Robust principal component analysis? Journal of the ACM 58 (3).

Chen, G., Lerman, G., 2009. Spectral curvature clustering (SCC). International Journal of Computer Vision 81 (3), 317-330.

Costeira, J., Kanade, T., 1998. A multibody factorization method for independently moving objects. International Journal of Computer Vision 29 (3), 159-179.

Elhamifar, E., Vidal, R., 2009. Sparse subspace clustering. In: IEEE Conference on Computer Vision and Pattern Recognition.

Elhamifar, E., Vidal, R., 2010. Clustering disjoint subspaces via sparse representation. In: IEEE International Conference on Acoustics, Speech, and Signal Processing.

Elhamifar, E., Vidal, R., 2013. Sparse subspace clustering: Algorithm, theory, and applications. IEEE Transactions on Pattern Analysis and Machine Intelligence.

Favaro, P., Vidal, R., Ravichandran, A., 2011. A closed form solution to robust subspace estimation and clustering. In: IEEE Conference on Computer Vision and Pattern Recognition.

Gear, C.W., 1998. Multibody grouping from motion images. Int. Journal of Computer Vision 29 (2), 133-150.

Goh, A., Vidal, R., 2007. Segmenting motions of different types by unsupervised manifold clustering. In: IEEE Conference on Computer Vision and Pattern Recognition.

Gruber, A., Weiss, Y., 2004. Multibody factorization with uncertainty and missing data using the EM algorithm. In: IEEE Conf. on Computer Vision and Pattern Recognition, vol. I, pp. 707-714.

Ho, J., Yang, M.H., Lim, J., Lee, K., Kriegman, D., 2003. Clustering appearances of objects under varying illumination conditions. In: IEEE Conference on Computer Vision and Pattern Recognition.

Hong, W., Wright, J., Huang, K., Ma, Y., 2006. Multi-scale hybrid linear models for lossy image representation. IEEE Trans. on Image Processing 15 (12), 3655-3671.

Lauer, F., Schnörr, C., 2009. Spectral clustering of linear subspaces for motion segmentation. In: IEEE International Conference on Computer Vision.

Lee, K.-C., Ho, J., Kriegman, D., 2005. Acquiring linear subspaces for face recognition under variable lighting. IEEE Transactions on Pattern Analysis and Machine Intelligence 27 (5), 684-698.

Lin, Z., Chen, M., Wu, L., Ma, Y., 2011. The augmented Lagrange multiplier method for exact recovery of corrupted low-rank matrices. arXiv:1009.5055v2.

Liu, G., Lin, Z., Yan, S., Sun, J., Yu, Y., Ma, Y., 2011. Robust recovery of subspace structures by low-rank representation. In: http://arxiv.org/pdf/1010.2955v1.

${ }^{1}$ One can also show that $\phi\left(\beta_{1}\left(\sigma_{1}\right), \sigma_{1}\right)<\phi\left(\beta_{3}\left(\sigma_{1}\right), \sigma_{1}\right)$ as follows:

$\phi\left(\beta_{1}\left(\sigma_{1}\right), \sigma_{1}\right)=\frac{\alpha \tau}{2} \frac{1}{\alpha+\tau} \frac{16}{9} \sqrt{\frac{3}{\alpha \tau}}=\frac{8 \tau}{3(\alpha+\tau)} \sqrt{\frac{\alpha}{3 \tau}}$

$\phi\left(\beta_{3}\left(\sigma_{1}\right), \sigma_{1}\right)=1-\frac{1}{2 \tau} \beta_{3}^{-2}+\frac{\alpha}{2}\left(\sigma-\beta_{3}\right)^{2}=1-\frac{1}{2 \tau} \beta_{3}^{-2}+\frac{\alpha}{18} \beta_{3}^{2}$

$=1-\frac{9-\alpha \tau \beta_{3}^{4}}{18 \tau \beta_{3}^{2}}=1-\frac{1}{3 \tau \beta_{3}^{2}}=1-\frac{1}{3} \sqrt{\frac{\alpha}{3 \tau}}$.

Therefore, $\phi\left(\beta_{1}\left(\sigma_{1}\right), \sigma_{1}\right)<\phi\left(\beta_{3}\left(\sigma_{1}\right), \sigma_{1}\right)$ because

$\frac{1}{3}\left(\frac{8 \tau}{\alpha+\tau}+1\right) \sqrt{\frac{\alpha}{3 \tau}}<1 \Longleftrightarrow\left(\frac{9 \tau+\alpha}{\alpha+\tau}\right)^{2} \frac{\alpha}{27 \tau}<1 \Longleftrightarrow(3 \tau-\alpha)^{3}>0$,

which follows from the fact that $3 \tau>\alpha$. 
Liu, G., Lin, Z., Yan, S., Sun, J., Yu, Y., Ma, Y., 2012. Robust recovery of subspace structures by low-rank representation. IEEE Transactions on Pattern Analysis and Machine Intelligence.

Liu, G., Lin, Z., Yu, Y., 2010. Robust subspace segmentation by low-rank representation. In: International Conference on Machine Learning.

Lu, L., Vidal, R., 2006. Combined central and subspace clustering on computer vision applications. In: International Conference on Machine Learning, pp. 593-600.

Ma, Y., Derksen, H., Hong, W., Wright, J., 2007. Segmentation of multivariate mixe data via lossy coding and compression. IEEE Transactions on Pattern Analysis and Machine Intelligence 29 (9), 1546-1562.

Mirsky, L., 1975. A trace inequality of John von Neumann. Monatshefte für Mathematic 79, 303-306.

Parikh, N., Boyd, S., 2013. Proximal Algorithms. Now.

Rao, S., Tron, R., Ma, Y., Vidal, R., 2008. Motion segmentation via robust subspace separation in the presence of outlying, incomplete, or corrupted trajectories. In: IEEE Conference on Computer Vision and Pattern Recognition.

Rao, S., Tron, R., Vidal, R., Ma, Y., 2010. Motion segmentation in the presence of outlying, incomplete, or corrupted trajectories. IEEE Transactions on Pattern Analysis and Machine Intelligence 32 (10), 1832-1845.

Recht, B., Fazel, M., Parrilo, P., 2010. Guaranteed minimum-rank solutions of linear matrix equations via nuclear norm minimization. SIAM Review 52 (3), 471-501.

Soltanolkotabi, M., Elhamifar, E., Candes, E., 2013. Robust subspace clustering. http://arxiv.org/abs/1301.2603.

Sugaya, Y., Kanatani, K., 2004. Geometric structure of degeneracy for multi-body motion segmentation. In: Workshop on Statistical Methods in Video Processing.

Tipping, M., Bishop, C., 1999. Mixtures of probabilistic principal component analyzers. Neural Computation 11 (2), 443-482.

Tomasi, C., Kanade, T., 1992. Shape and motion from image streams under orthography: A factorization method. International Journal of Computer Vision 9, 137-154.

Tron, R., Vidal, R., 2007. A benchmark for the comparison of 3-D motion segmentation algorithms. In: IEEE Conference on Computer Vision and Pattern Recognition.
Tseng, P., 2000. Nearest q-flat to m points. Journal of Optimization Theory and Applications 105 (1), 249-252.

Tseng, P., 2001. Convergence of a block coordinate descent method for nondifferentiable minimization. Journal of Optimization Theory and Applications 109 (3), 475-494.

Vidal, R., 2011. Subspace clustering. IEEE Signal Processing Magazine 28 (3), 52-68.

Vidal, R., Ma, Y., Piazzi, J., 2004. A new GPCA algorithm for clustering subspaces by fitting, differentiating and dividing polynomials. In: IEEE Conference on Computer Vision and Pattern Recognition, vol. I, pp. 510-517.

Vidal, R., Ma, Y., Sastry, S., 2003a. Generalized Principal Component Analysis (GPCA). In: IEEE Conference on Computer Vision and Pattern Recognition, vol. I, pp. 621-628.

Vidal, R., Ma, Y., Sastry, S., 2005. Generalized Principal Component Analysis (GPCA). IEEE Transactions on Pattern Analysis and Machine Intelligence 27 (12), 1-15.

Vidal, R., Soatto, S., Ma, Y., Sastry, S., 2003b. An algebraic geometric approach to the identification of a class of linear hybrid systems. In: Conference on Decision and Control, pp. 167-172.

Vidal, R., Tron, R., Hartley, R., 2008. Multiframe motion segmentation with missing data using PowerFactorization and GPCA. International Journal of Computer Vision 79 (1), 85-105.

von Luxburg, U., 2007. A tutorial on spectral clustering. Statistics and Computing 17.

Yan, J., Pollefeys, M., 2006. A general framework for motion segmentation: Independent, articulated, rigid, non-rigid, degenerate and non-degenerate. In: European Conf. on Computer Vision, pp. 94-106.

Yang, A., Wright, J., Ma, Y., Sastry, S., 2008. Unsupervised segmentation of natural images via lossy data compression. Computer Vision and Image Understanding 110 (2), 212-225.

Yang, A.Y., Rao, S., Ma, Y., 2006. Robust statistical estimation and segmentation of multiple subspaces. In: Workshop on 25 years of RANSAC.

Zhang, T., Szlam, A., Lerman, G., 2009. Median $k$-flats for hybrid linear modeling with many outliers. In: Workshop on Subspace Methods.

Zhang, T., Szlam, A., Wang, Y., Lerman, G., 2010. Hybrid linear modeling via local best-fit flats. In: IEEE Conference on Computer Vision and Pattern Recognition, pp. 1927-1934. 